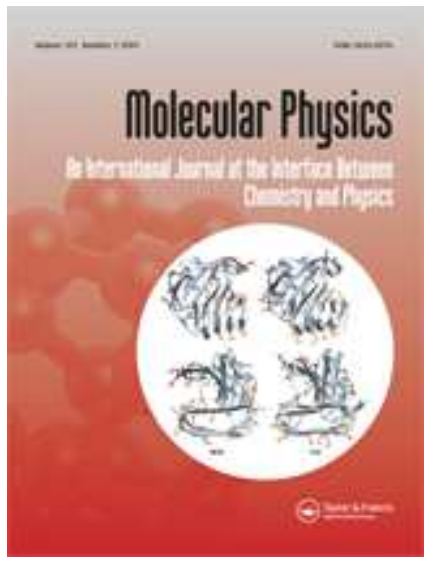

\title{
BINDING He ATOMS TO HYDROGEN MOIETIES: QUANTUM FEATURES FROM ULTRAWEAK INTERACTIONS
}

\begin{tabular}{|c|c|}
\hline Journal: & Molecular Physics \\
\hline Manuscript ID: & TMPH-2009-0334.R1 \\
\hline Manuscript Type: & Full Paper \\
\hline $\begin{array}{r}\text { Date Submitted by the } \\
\text { Author: }\end{array}$ & 11-Nov-2009 \\
\hline Complete List of Authors: & $\begin{array}{l}\text { orlandini, sergio; Univerisy of Rome 1, chemistry } \\
\text { Coccia, Emanuele; University of Rome "Sapienza", Chemistry } \\
\text { Baccarelli, Isabella; CASPUR, super computing consortium } \\
\text { Gianturco, F; University of Rome, chemistry } \\
\text { Garrido, Eduardo; Consejo superior de investigaciones cientificas, } \\
\text { Instituto de estructura de la materia } \\
\text { Gonzalez-Lezana, Tomas; Consejo superior de investigaciones } \\
\text { cientificas, Instituto de fisica fundamental } \\
\text { Delgado-Barrio, Gerardo; CSIC, IMAFF } \\
\text { Villarreal, Pablo; CSIC, IMAFF }\end{array}$ \\
\hline Keywords: & bound states, weakly interacting trimers, Hydrogen moieties \\
\hline \multicolumn{2}{|c|}{$\begin{array}{l}\text { Note: The following files were submitted by the author for peer review, but cannot be converted } \\
\text { to PDF. You must view these files (e.g. movies) online. }\end{array}$} \\
\hline gianturco.zip & \\
\hline
\end{tabular}




\section{$\$$ Manuscript Central}




\title{
BINDING He ATOMS TO HYDROGEN MOIETIES: QUANTUM FEATURES FROM ULTRAWEAK INTERACTIONS
}

\author{
S. Orlandini ${ }^{\ddagger}$, E. Cocciał, I. Baccarelli ${ }^{\ddagger}$, and F.A. Gianturco $\dagger^{*}$ \\ $\dagger$ Department of Chemistry and CNISM, University of Rome La Sapienza, \\ Piazzale A. Moro 5, 00185 Rome, Italy \\ ${ }^{\ddagger}$ CASPUR, Supercomputing Consortium, via dei Tizii 6, 00185 Rome, Italy \\ E. Garrido \\ Instituto de Estructura de la Materia, \\ Consejo Superior de Investigaciones Cientificas, Serrano 123, 28006 Madrid, Spain \\ T. González-Lezana, G. Delgado-Barrio and P. Villarreal \\ Instituto de Física Fundamental, Consejo Superior de \\ Investigaciones Científicas, Serrano 123, 28006 Madrid, Spain
}

\footnotetext{
* Corresponding author; e.mail address: fa.gianturco@caspur.it; fax: +39.06.49913305.
} 


\begin{abstract}
The possible existence of bound states in small helium clusters containing atomic and molecular hydrogen has been investigated by means of three completely independent sets of calculations. The first set employs the Jacobi-Davidson filtering procedure recently implemented in the Distributed Gaussian Functions (DGF) approach, the second one is based on the solution of the Faddeev equation by means of the hyperspherical adiabatic expansion method and the third follows the Quantum Monte Carlo approach. The partners within each complex are taken to interact via two-body potentials chosen among the most accurate existing in the literature. All the present quantum treatments show that no bound states are found for $\mathrm{H}\left({ }^{4} \mathrm{He}\right)_{2}$ and for $\mathrm{D}\left({ }^{4} \mathrm{He}\right)_{2}$ while only one very diffuse bound state is present in $\mathrm{T}\left({ }^{4} \mathrm{He}\right)_{2}$, where $\mathrm{H}, \mathrm{D}$ and $\mathrm{T}$ are the three isotopes of hydrogen. The substitution of the atomic dopant with its molecular counterpart, $\mathrm{H}_{2}$, also gives rise to only one bound state whose spatial attributes are reported and analysed, underlining again the ultraweak nature of the interaction forces and the need to implement highly specific tools for the investigation of the ensuing bound states.
\end{abstract}




\section{INTRODUCTION}

In comparison with the many molecular systems which are held together chiefly by Van der Waals (VdW) forces, those which involve one or more helium atoms as partners have certainly received a great deal of attention, in the last twenty years or so, both experimentally and from the theoretical standpoint [1-4]. Through the extensive analysis and observations which have been carried out on a great variety of such systems, in fact, our cumulative knowledge of their behavior has made considerable progress: from our better understanding of the superfluid behavior of large scale systems with a degree of coherence, to the contributions of He-containing gases for the occurrence of Bose-Einstein condensation (BEC), just to cite two of the more intriguing physical effects $[5,6]$. It has also been particularly interesting the general capability found in He aggregates, from small clusters to large droplets, to enclose and interact with a broad variety of molecular systems, neutral and ionized, which turned out to be only very slightly perturbed by the presence of this quantum fluid that surrounds the molecules [4]. It therefore becomes of prime interest to provide and collect as much data as possible on the quantitative knowledge of the strength and extension of the weak $\mathrm{VdW}$ forces which act within cluster structures that contain a variable number of helium atoms, generally in the bosonic variant, and an atomic or molecular dopant. In order to make progress in this direction one needs to be able to describe as realistically as possible their spatial arrangements, invariably characterized by very strongly anharmonic internal motions and by large values of their zero-point energies [7].

One important computational consequence of this property is the increased difficulty in obtaining numerically converged results for both eigenvalues and eigenfunctions of these systems [8]. Hence, the search of bound states due to such ultra-weak forces represents a challenging test-bed for any computational approach: for example, a method which is perfectly suited to the calculation of bound states in ordinary, chemically-bound complexes, can fail with systems requiring extended integration ranges. It is thus mandatory to compare as much as possible the findings obtained with different theoretical and computational procedures whose reliability, though already assessed for the study of more strongly bound systems, needs to be confirmed for the study of ultra-weakly bound VdW species, while the accurate assessment of the behaviour of small, three-body (3B) clusters will help us to guide further calculations directed at larger, mixed clusters containing He or $\mathrm{H}$ [9]. 
One of the cases in point involves the study of a three-particle system which contains two ${ }^{4} \mathrm{He}$ atoms, well known to be only very weakly bound to each other [10], attached to the lighter hydrogen atom, which is known not to be bound to ${ }^{4} \mathrm{He}$ [11]. One would in fact expect that three-particle aggregates which are made up of such weakly bound partners should present either no bound states or at most very floppy bound states where the partner atoms are highly delocalized in space. The $\mathrm{H}_{-}{ }^{4} \mathrm{He}_{2}$ system has been already studied a while ago by Lin and $\mathrm{Li}$ [12], who searched for the existence of weakly bound molecules like He-He-H and He-H-H. With the atom-atom potentials which they used, they indeed found no bound states for the $\mathrm{H}^{4}{ }^{4} \mathrm{He}_{2}$ system. The same result has been confirmed by the more recent calculations of Ref. [8]. In the present paper we investigate, first of all, if the H-He interaction is capable of sustaining any bound state and then analyse the effects on the existence of bound states caused by small changes like the dopant's isotopic substitution, the increase of the number of helium atoms and the tuning of the dopant-solvent interaction. The latter is simulated by substituting the atomic dopant with its molecular counterpart $\mathrm{H}_{2}$ treated as a structureless atomic species $(2 \mathrm{H})$ with mass equal to the deuterium isotope and interacting with the He atoms via an approximate isotropic potential which originates from the correct $\mathrm{He}-\mathrm{H}_{2}$ potential. One should further note that indeed earlier publications have already been devoted to ${ }^{4} \mathrm{He}$ clusters containing a single $\mathrm{H}_{2}$ impurity. Variational Monte Carlo (VMC) calculations on ${ }^{4} \mathrm{He}$ clusters with a single $\mathrm{H}_{2}$ [13] indicate that the $\mathrm{H}_{2}$ is delocalized, moving almost freely from the inside to the outside of the cluster. On the other hand, results reported by Gordillo in Ref. [14] on ${ }^{4} \mathrm{He} / \mathrm{p}-\mathrm{H}_{2}$ binary clusters, which are obtained by Path Integral Monte Carlo simulations, show that the hydrogen tends to be located in the inner regions of the cluster. Recently, Toennies and co-workers identified the $\mathrm{H}_{2}-{ }^{4} \mathrm{He}$ van der Waals complex in a molecular beam by a diffraction experiment, using a $100 \mathrm{~nm}$ period transmission grating [15], a result confirmed by our later computational findings of a bound state for that system [16]. In that paper, the $\mathrm{p}-\mathrm{H}_{2}$ molecule interacts with one helium atom, be it either ${ }^{4} \mathrm{He}$ or ${ }^{3} \mathrm{He}[16]$. We had found that a bound "halo" state indeed exists for both variants of the helium atom and further established that the very mild orientational features of the $\mathrm{H}_{2}{ }^{-}{ }^{4} \mathrm{He}$ potential yielded very similar results when the molecular partner was treated as a "spherical" $(2 \mathrm{H})$ partner species [16].

A more extensive Quantum Monte Carlo (QMC) study has been further reported in our recent work [9] on the $\mathrm{H}_{2}\left({ }^{4} \mathrm{He}\right)_{N}$ system, where we carried VMC and Diffusion Monte 
Carlo (DMC) calculations in order to extract nanoscopic information about energetics and geometrical features of these very weakly bound clusters; we used the fully anisotropic $\mathrm{H}_{2}$ ${ }^{4} \mathrm{He}$ PES [17] as one of the "two-body" (2B) potentials in the clusters and we found there that the calculations indicate the impurity to be bound to the solvent and no shell structuring is present across the size range which we have examined ( $N$ from 1 to 100). The emerging physical picture is one in which the dopant is progressively embedded within the cluster as $N$ increases, although it does not initially reside at the exact center of the droplet, in substantial agreement with the findings of Ref. [14].

In the present work three completely independent sets of calculations have been employed to quantitatively assess the existence of bound states and, when existing, of their spatial attributes for the rotationless $\mathrm{H}\left({ }^{4} \mathrm{He}\right)_{2}$ system and its isotopic variants, $\mathrm{D}\left({ }^{4} \mathrm{He}\right)_{2}$ and $\mathrm{T}\left({ }^{4} \mathrm{He}\right)_{2}$. The first method employs the Jacobi-Davidson (JD) filtering procedure recently implemented [18] in the Distributed Gaussian Functions (DGF) approach, introduced a few years ago to especially treat very floppy triatomic systems [19-22]. The second approach is based on the solution of the Faddeev equation by means of the hyperspherical adiabatic expansion method [23]. The third tool follows the QMC scheme and is also employed to study the next larger cluster with three helium atoms. It will be shown that all methods provide the same, converged answers on the existence or non-existence of bound states in the above clusters and further supply us with a detailed description of the spatial features of "molecular" $\mathrm{T}\left({ }^{4} \mathrm{He}\right)_{2}$, the only one presenting a bound state.

We additionally investigate the effect of substituting the atomic impurity $\mathrm{H}$ with its molecular counterpart $\mathrm{H}_{2}$, using the DGF and DMC methods; they will be shown to exhibit excellent agreement both on the energetics and on the spatial characterization of the only bound state found for $\mathrm{H}_{2}\left({ }^{4} \mathrm{He}\right)_{2}$.

The paper is organized as follows: next Section II outlines the three approaches and describes their present implementation, while Section III reports our results. Our discussion and present conclusions are given in the final Section IV. For the sake of clarity, in the following ${ }^{4} \mathrm{He}$ will become He. 


\section{THE THEORETICAL METHODS}

\section{A. IMPLEMENTING THE DGF METHOD}

The very weak nature of the interaction forces between the three particles of the present study allows us to describe the total potential energy surface as a sum of the $2 \mathrm{~B}$ potentials $\left(V\left(R_{i}\right)\right)$. The Hamiltonian can be written in terms of atom-atom coordinates as

$$
H\left(R_{1}, R_{2}, R_{3}\right)=T\left(R_{1}, R_{2}, R_{3}\right)+\sum_{i=1}^{3} V\left(R_{i}\right)
$$

where $T\left(R_{1}, R_{2}, R_{3}\right)$ is the kinetic operator for the $\mathrm{AB}_{2}$ triatomic system and has been given explicitly many times before $[22,24]$.

The total wave function (WF) of the $K$-th state is expanded in terms of basis functions $\phi_{j}\left(R_{1}, R_{2}, R_{3}\right)$

$$
\Phi^{(K)}\left(R_{1}, R_{2}, R_{3}\right)=\sum_{j} a_{j}^{(K)} \phi_{j}\left(R_{1}, R_{2}, R_{3}\right)
$$

Each basis function is defined as a correctly symmetrized product of three DGFs centered along the three atom-atom coordinates and, in the case of a system with two identical particles, can be written as:

$$
\phi_{j}\left(R_{1}, R_{2}, R_{3}\right)=N_{l m n}^{-1 / 2} \sum_{P \in S_{2}} P\left[\varphi_{l}\left(R_{1}-R_{l}\right) \varphi_{m}\left(R_{2}-R_{m}\right)\right] \varphi_{n}\left(R_{3}-R_{n}\right) .
$$

In the above expression $\varphi_{p}\left(R_{i}-R_{p}\right)$ is a Gaussian function along the $R_{i}$ coordinate and centered in the $R_{p}$ point, as suggested by Hamilton et al. [25], $N_{l m n}$ is a normalization constant expressed in terms of overlap integrals between the Gaussian functions [24], and $j$ denotes a collective index such as $j=(l \leq m ; n)$ for the case of two identical atoms. We can now exploit the normalization condition of the total WF for the $K$-th bound state to define a sort of weight, $P_{j}^{(K)}$, associated to each basis function $\phi_{j}$

$$
1=\left\langle\Phi^{(K)} \mid \Phi^{(K)}\right\rangle=\sum_{j} a_{j}^{(K)}\left\langle\Phi^{(K)} \mid \phi_{j}\right\rangle=\sum_{j} P_{j}^{(K)}
$$

We will refer to the $P_{j}^{(K)}$ as to pseudo-weights (PWs) since, although their sum is equal to one, their character may also be negative. Via these PWs, the moments of a given quantity $x^{n}$, depending on the three bond coordinates, can be calculated resorting to the mean value 
theorem:

$$
\left\langle x^{n}\right\rangle^{(K)}=\sum_{j} a_{j}^{(K)}\left\langle\Phi^{(K)}\left|x^{n}\right| \phi_{j}\right\rangle \approx \sum_{j} P_{j}^{(K)} x_{j}^{n}
$$

where in the integrals involved we have assumed that the magnitude $x^{n}$ has been replaced by its mean value, $x_{j}^{n}$, for the triangular configuration $\phi_{j}$. With this procedure quantities such as the atom-atom distances, the area, the cosine values, can be evaluated to obtain the most probable geometries of the corresponding bound states. We can also define the weight of each type of triangular family (scalene, collinear, flat or tall isosceles and equilateral) by summing the PWs $P_{j}^{(K)}$ associated to each element of the family.

Pair distributions in the $R_{i}$ coordinates are evaluated from the total WF of Eq. (2) as:

$$
\mathcal{D}_{D G F}^{(K)}\left(R_{i}\right)=\iint d R_{j} d R_{k}\left|\Phi^{(K)}\left(R_{i}, R_{j}, R_{k}\right)\right|^{2}
$$

In order to minimize the unphysical behaviour of the total WFs at the triangular boundaries, we require for each product of three Gaussian functions $\varphi_{l} \varphi_{m} \varphi_{n}$ which belongs to the basis set that the DGF centers, $R_{p}$, satisfy the triangle requirement (TIR) in the more restrictive way $\left|R_{l}-R_{m}\right|<R_{n}<R_{l}+R_{m}$.

\section{The "quality" indicator $\mathcal{W}$}

An important indicator of the quality of the DGF bases is the "badness" operator $(\mathcal{W})$ $[20,26]$ which quantifies the relevance of the subdomain where the total WF is unphysically non-zero

$$
\mathcal{W}\left(R_{1}, R_{2}, R_{3}\right)= \begin{cases}0, & \left|R_{1}-R_{2}\right|<R_{3}<R_{1}+R_{2} \text { holds } \\ 1, & \text { otherwise. }\end{cases}
$$

The average value $\langle\mathcal{W}\rangle$ estimates how much the norm of the WF integrated over the entire space (equal to unity) differs from the norm integrated only over the domain where the TIR is satisfied. This control indicator measures the quality of the representation of each bound state of the trimer in the chosen basis set.

In Ref. [26] we found that the best locations of the $N$ DGF centers are given by the following formula

$$
R_{i}=n \cdot \Delta+i \cdot \Delta, \quad i=0,1,2 \ldots N-1
$$


where the value of $n$ provides the correct multiple of $\Delta$ that locates the radial position of the first DGF of the expansion set. However, some residual pathological behaviour could still affect the computed bound energy value whenever linear arrangements significantly contribute to the description of that bound state. In order to overcome this limitation, we set up an extrapolation procedure [27] which allows us to produce final results completely unaffected by pathological contributions, i.e., with zero badness. We repeat the bound-state calculation by shifting the positions of the DGF centers, thereby establishing the dependence of the energetics on the badness quantity, which is then fitted to a polynomial functional finally extrapolated to zero badness. A second extrapolation with respect to the step $\Delta$ is now carried out in order to reach the $\delta$-function limit of the initial basis set. The actual functional expressions were different in the two cases. To reach zero badness we employed the functional form:

$$
E_{\Delta}^{e x}(x)=a_{0}+a_{1} x^{2}+a_{2} x^{4}+a_{3} x^{6}
$$

where $x=\langle\mathcal{W}\rangle$. To reach the $\delta$-function limit of zero width we have used

$$
E_{\delta}^{e x}(x)=a_{0}+a_{1} x^{2}+a_{2} x^{a_{3}}
$$

where $x=\Delta$. In the examples of this work the extrapolation of Eq. 9 typically produced a $\chi^{2}$ value of $9 \times 10^{-12}$ and a correlation coefficient of $1 \times 10^{-6}$. The extrapolation at zerowidth $\delta$-function has been achieving a $\chi_{\delta}^{2}$ value of $3.7 \times 10^{-9}$ and a correlation coefficient of 0.99997. All parameters of Eqs. 9 and 10 are available on request.

\section{The Jacobi-Davidson filtering procedure}

Since the basis functions given by Eq.(3) are not orthogonal one needs to solve a generalized matrix eigenproblem which involves the overlap matrix of the basis set. Due to the sparse nature and the huge size of the overlap and Hamiltonian matrices, it is convenient to use an iterative method for the solution of the eigenproblem, such as the JD procedure [28] that we recently implemented within our DGF approach [18]. The details of the implementation have been described before [18] and therefore only a brief outline shall be given below.

Our procedure begins with the generation of a search subspace spanned by an orthonormal basis $\left\{v_{1}, \ldots, v_{k}\right\}$ starting from a randomly chosen non trivial vector; by imposing the 
Galerkin condition [29] we then obtain a test subspace spanned by another orthonormal basis $\left\{w_{1}, \ldots, w_{k}\right\}$.

The reduced problem takes the form: $\left(\mathbf{W}_{k}^{*} \mathbf{H} \mathbf{V}_{k}\right) y=\theta\left(\mathbf{W}_{k}^{*} \mathbf{S} \mathbf{V}_{k}\right) y$, where $\mathbf{V}_{k}$ and $\mathbf{W}_{k}$ denote the $n \times k$ matrices with the basis vectors as columns and $(\theta, y)$ are the solutions of the reduced eigenproblem. The Ritz values $\theta$ and the Ritz vectors $u=\mathbf{V}_{k} y$ are approximations to the original eigenpair. The reduced eigenproblem is now of much smaller dimensions since $k \ll n$.

In order to improve the quality of the subspaces we solve the JD correction equation, which is given by

$$
\left(\mathbf{I}-z z^{*}\right)(\mathbf{H}-\theta \mathbf{S})\left(\mathbf{I}-u u^{*}\right) u^{\perp}=-r \equiv-(\mathbf{H}-\theta \mathbf{S}) u
$$

in which $u^{\perp}$ is the orthogonal correction to the approximation of the eigenvector and $z$ is an auxiliary vector given by $z=(\mathbf{H}+\theta \mathbf{S})$.

The JD method has been implemented in practice by applying a variant of the QZalgorithm [30]. A partial generalized Schur decomposition is made for the projected matrices $\mathbf{W}_{k}^{*} \mathbf{H} \mathbf{V}_{k}$ and $\mathbf{W}_{k}^{*} \mathbf{S V}_{k}$, so that we find orthogonal matrices $\mathbf{U}_{\mathbf{L}}$ and $\mathbf{U}_{\mathbf{R}}$ which satisfy

$$
\begin{aligned}
& \mathbf{U}_{\mathbf{L}}^{*}\left(\mathbf{W}_{k}^{*} \mathbf{H} \mathbf{V}_{k}\right) \mathbf{U}_{\mathbf{R}}=\mathbf{S}_{\mathbf{A}} \\
& \mathbf{U}_{\mathbf{L}}^{*}\left(\mathbf{W}_{k}^{*} \mathbf{S} \mathbf{V}_{k}\right) \mathbf{U}_{\mathbf{R}}=\mathbf{S}_{\mathbf{B}}
\end{aligned}
$$

where $\mathbf{S}_{\mathbf{A}}$ and $\mathbf{S}_{\mathbf{B}}$ are upper triangular matrices. The approximation of the eigenpair becomes $\theta=\mathbf{S}_{\mathbf{A}}(1,1) / \mathbf{S}_{\mathbf{B}}(1,1)$ and $u=\mathbf{V}_{k} \mathbf{U}_{\mathbf{R}}(i, 1)$ with $i=1, \ldots, k$.

\section{B. THE FADDEEV EQUATIONS}

3B systems are often described by use of the so called Jacobi coordinates, which are defined as:

$$
\begin{aligned}
\mathbf{x}_{i}=\mu_{j k} \mathbf{r}_{j k}, & \mathbf{r}_{j k}=\mathbf{r}_{j}-\mathbf{r}_{k} \\
\mathbf{y}_{i}=\mu_{i(j k)} \mathbf{r}_{i(j k)}, & \mathbf{r}_{i(j k)}=\mathbf{r}_{i}-\frac{m_{j} \mathbf{r}_{j}+m_{k} \mathbf{r}_{k}}{m_{j}+m_{k}}, \\
\mu_{j k}=\left(\frac{1}{m} \frac{m_{j} m_{k}}{m_{j}+m_{k}}\right)^{1 / 2}, & \mu_{i(j k)}=\left(\frac{1}{m} \frac{m_{i}\left(m_{j}+m_{k}\right)}{m_{1}+m_{2}+m_{3}}\right)^{1 / 2},
\end{aligned}
$$

where $\{i, j, k\}$ is a cyclic permutation of $\{1,2,3\}, m_{k}$ and $\mathbf{r}_{k}$ are the mass and coordinate of particle $k$, and $\mu^{2}$ are the reduced masses of the subsystems in units of an arbitrary 
normalization mass $m$. The $\mathbf{x}_{i}$ and $\mathbf{r}_{j k}$ coordinates correspond to the interparticle distance between the two He atoms when $i=1$ and to the $\mathrm{He}-\mathrm{H}$ distance when $i=2,3$. In the former case, $\mathbf{y}_{i}$ and $\mathbf{r}_{i(j k)}$ are set along the direction which connects the $\mathrm{H}$ atom and the center of mass of the $\mathrm{He}_{2}$ unit, whereas for the $i=2,3$ arrangements, they correspond to the distance between one of the He atom and the center of mass of $\mathrm{He}-\mathrm{H}$.

Obviously three different sets of Jacobi coordinates $\left\{\mathbf{x}_{i}, \mathbf{y}_{i}\right\}$ are possible, and in principle any of them can be chosen for a correct description of the system. However, in some cases, the particular properties of the intrinsic $2 \mathrm{~B}$ subsystems can make one of the Jacobi sets more suitable than the others. A clear example is the one in which one of the $2 \mathrm{~B}$ subsystems has a bound state. The Jacobi set where the $\mathbf{x}$ coordinate is defined between these two particles is more appropriate, since they are the natural coordinates describing the asymptotics of the $3 \mathrm{~B}$ WF. For the same reason, 3B systems where more than one of the inner 2B subsystems has a bound state (or a low-lying resonance) are difficult to describe with a single set of Jacobi coordinates. For these cases a better choice is to use democratic coordinates providing a completely symmetric treatment of each Jacobi set. This is achieved by writing the total $3 \mathrm{~B}$ WF for a total angular momentum $J$ and its corresponding projection along the space-fixed Z-axis, $M$, of as:

$$
\Psi^{J M}=\sum_{i=1}^{3} \psi_{(i)}^{J M}\left(\mathbf{x}_{i}, \mathbf{y}_{i}\right),
$$

where each of the components $\psi_{(i)}^{J M}\left(\mathbf{x}_{i}, \mathbf{y}_{i}\right)$ satisfies the equation:

$$
(T-E) \psi_{(i)}^{J M}\left(\mathbf{x}_{i}, \mathbf{y}_{i}\right)+V_{j k}\left(\psi_{(i)}^{J M}\left(\mathbf{x}_{i}, \mathbf{y}_{i}\right)+\psi_{(j)}^{J M}\left(\mathbf{x}_{j}, \mathbf{y}_{j}\right)+\psi_{(k)}^{J M}\left(\mathbf{x}_{k}, \mathbf{y}_{k}\right)\right)=0
$$

where $T$ is the kinetic energy operator, $V_{j k}$ is the interaction between particles $j$ and $k$, and $E$ is total 3B energy. A cyclic permutation of the indexes $\{i, j, k\}$ in Eq.(16) gives rise to the three Faddeev equations, which after summation provide the usual Schrödinger equation.

\section{The hyperspherical adiabatic expansion method}

From the three sets of Jacobi coordinates $\left\{\mathbf{x}_{i}, \mathbf{y}_{i}\right\}$ we construct the corresponding three sets of hyperspherical coordinates $\left\{\rho, \Omega_{i}\right\} \equiv\left\{\rho, \alpha_{i}, \Omega_{x_{i}}, \Omega_{y_{i}}\right\}$ where $\Omega_{x_{i}}$ and $\Omega_{y_{i}}$ give the directions of $\mathbf{x}_{i}$ and $\mathbf{y}_{i}$, and the hyperradius $\rho$ and hyperangle $\alpha_{i}$ are defined by

$$
x_{i}=\rho \sin \alpha_{i}, \quad y_{i}=\rho \cos \alpha_{i},
$$


in terms of which the volume element is given by $\rho^{5} \mathrm{~d} \Omega_{i} \mathrm{~d} \rho$, where $\mathrm{d} \Omega_{i}=\sin ^{2} \alpha_{i} \cos ^{2} \alpha_{i} \mathrm{~d} \alpha_{i} \mathrm{~d} \Omega_{x_{i}} \mathrm{~d} \Omega_{y_{i}}$.

The kinetic energy operator is then written as

$$
\begin{array}{r}
T=\frac{\hbar^{2}}{2 m}\left(-\rho^{-5 / 2} \frac{\partial^{2}}{\partial \rho^{2}} \rho^{5 / 2}+\frac{15}{4 \rho^{2}}+\frac{\hat{\Lambda}_{i}^{2}}{\rho^{2}}\right), \\
\hat{\Lambda}_{i}^{2}=-\frac{1}{\sin \left(2 \alpha_{i}\right)} \frac{\partial^{2}}{\partial \alpha_{i}^{2}} \sin \left(2 \alpha_{i}\right)+\frac{\hat{l}_{x_{i}}^{2}}{\sin ^{2} \alpha_{i}}+\frac{\hat{l}_{y_{i}}^{2}}{\cos ^{2} \alpha_{i}}-4,
\end{array}
$$

where the angular momentum operators $\hat{l}_{x_{i}}^{2}$ and $\hat{l}_{y_{i}}^{2}$ are related to the $\mathbf{x}_{\mathbf{i}}$ and $\mathbf{y}_{\mathbf{i}}$ degrees of freedom.

As detailed in Ref. [23], each of the components $\psi_{(i)}^{J M}$ in the total WF (15) is now expanded in terms of a complete set of generalized angular functions $\phi_{n}^{(i) J M}\left(\rho, \Omega_{i}\right)$ :

$$
\psi_{(i)}^{J M}\left(\mathbf{x}_{i}, \mathbf{y}_{i}\right)=\frac{1}{\rho^{5 / 2}} \sum_{n} f_{n}(\rho) \phi_{n}^{(i) J M}\left(\rho, \Omega_{i}\right)
$$

where $\rho^{-5 / 2}$ is the radial phase space factor and $n(=1,2,3 \ldots)$ labels the angular functions. The total 3B WF can then be written as:

$$
\Psi^{J M}=\frac{1}{\rho^{5 / 2}} \sum_{n} f_{n}(\rho) \Phi_{n}^{J M}\left(\rho, \Omega_{i}\right)
$$

where $\Phi_{n}^{J M}(\rho, \Omega)=\sum_{i} \phi_{n}^{(i) J M}$. The angular functions $\phi_{n}^{(i) J M}$ are expanded in terms of a complete basis set of hyperspherical harmonics, $\mathbf{Y}_{\ell_{x} \ell_{y}}^{\mathcal{K} J M}$, as [31]:

$$
\phi_{n}^{(i) J M}\left(\rho, \Omega_{i}\right)=\sum_{\mathcal{K} \ell_{x} \ell_{y}} C_{n \mathcal{K} \ell_{x} \ell_{y}}^{(i)}(\rho) \mathbf{Y}_{\ell_{x} \ell_{y}}^{\mathcal{K} J M}\left(\alpha_{i}, \Omega_{x_{i}}, \Omega_{y_{i}}\right),
$$

where $\mathcal{K}$ is the hypermoment, $\ell_{x}$ and $\ell_{y}$ are the orbital angular momenta associated with $\mathbf{x}$ and $\mathbf{y}$, respectively, and $C$ are the expansion coefficients. $\mathcal{K}$ is related to the $\ell_{x}$ and $\ell_{y}$ momenta as $\mathcal{K}=2 \nu+\ell_{x}+\ell_{y}$, where the $\nu$ index is varied in the calculation.

The adiabatic approach assumes that the hyperangles vary much more quickly than the radial coordinate $\rho$. After substitution of Eqs.(18) and (20) into the Faddeev equations (16), it is then possible to solve them first for a set of fixed values of $\rho$, in such a way that the angular functions (the adiabatic basis) are now, for each chosen $\rho$, as the eigenfunctions of the angular part of the Faddeev equations:

$$
\frac{\hbar^{2}}{2 m} \frac{1}{\rho^{2}}\left(\hat{\Lambda}^{2}-\lambda_{n}(\rho)\right) \phi_{n}^{(i) J M}+V_{j k}\left(\phi_{n}^{(i) J M}+\phi_{n}^{(j) J M}+\phi_{n}^{(k) J M}\right)=0
$$


where $\{i, j, k\}$ is a cyclic permutation of $\{1,2,3\}$. The eigenvalue problem given in the equation above is solved after expansion of the angular eigenfunctions in terms of hyperspherical harmonics (see [23] for details).

In a second step, the radial coefficients $f_{n}(\rho)$ in the expansion (15) are finally obtained from a coupled set of "radial" differential equations [23], i.e.

$$
\left(-\frac{\partial^{2}}{\partial \rho^{2}}-\frac{2 m E}{\hbar^{2}}+\frac{1}{\rho^{2}}\left(\lambda_{n}(\rho)+\frac{15}{4}\right)-Q_{n n}\right) f_{n}(\rho)=\sum_{n^{\prime} \neq n}\left(2 P_{n n^{\prime}} \frac{\partial}{\partial \rho}+Q_{n n^{\prime}}\right) f_{n^{\prime}}(\rho)
$$

where $E$ is the $3 \mathrm{~B}$ energy, the eigenvalues $\lambda_{n}(\rho)$ of the angular equations (23) enter as effective potentials, and the functions $P$ and $Q$ are defined as the angular integrals:

$$
\begin{aligned}
P_{n n^{\prime}}(\rho) & \equiv \int d \Omega \Phi_{n}^{J M *}(\rho, \Omega) \frac{\partial}{\partial \rho} \Phi_{n^{\prime}}^{J M}(\rho, \Omega), \\
Q_{n n^{\prime}}(\rho) & \equiv \int d \Omega \Phi_{n}^{J M *}(\rho, \Omega) \frac{\partial^{2}}{\partial \rho^{2}} \Phi_{n^{\prime}}^{J M}(\rho, \Omega) .
\end{aligned}
$$

Different properties of these coupling terms can be found for instance in [23].

The pair distribution, in terms of one or two of the coordinates defined in Eq. (13) for the arrangement $i$, can be calculated as:

$$
\begin{aligned}
\mathcal{D}_{\text {Faddeev }}^{(i)}\left(r_{j k}\right) & =\mu_{j k} \iiint y_{i}^{2} d y_{i} d \Omega_{y_{i}} x_{i}^{2} d \Omega_{x_{i}}\left|\psi_{(i)}^{J M}\left(\mathbf{x}_{i}, \mathbf{y}_{i}\right)\right|^{2} \\
D_{\text {Faddeev }}^{(i)}\left(r_{j k}, r_{i(j k)}\right) & =\mu_{j k} \mu_{i(j k)} \iint x_{i}^{2} d \Omega_{x} y_{i}^{2} d \Omega_{y}\left|\psi_{(i)}^{J M}\left(\mathbf{x}_{i}, \mathbf{y}_{i}\right)\right|^{2}
\end{aligned}
$$

The hyperspherical adiabatic expansion method sketched in this section has proved to be a suitable method to describe spatially extended and weakly bound 3B systems. In particular it has been frequently, and successfully, used to describe the structures of the so-called halo nuclei, like ${ }^{6} \mathrm{He}$ or ${ }^{11} \mathrm{Li}[32-35]$, as well as many other nuclei $\left({ }^{6} \mathrm{Li},{ }^{6} \mathrm{Be},{ }^{12} \mathrm{Be}\right.$, ${ }^{17} \mathrm{Ne}$ or ${ }^{12} \mathrm{C}$, among others) that can be treated as $3 \mathrm{~B}$ systems [36-40]. This method is able to accurately reproduce the asymptotic behaviour of the WFs, as tested for the analysis of Efimov effects [41-43], where an accurate computation of the WFs at very large distances is essential [44].

\section{QUANTUM MONTE CARLO METHODS}

The QMC approach for calculating the bound states of a weakly bound system can be divided into two main steps: the optimization of the trial WF $\Psi_{T}$ through a minimization 
procedure, implemented here in a VMC code [45], and the use of $\Psi_{T}$ to solve by a random walk the imaginary-time dependent Schrödinger equation in its importance sampling form:

$$
\left.-\frac{\partial f(\boldsymbol{R}, \tau)}{\partial \tau}=-D \nabla^{2} f(\boldsymbol{R}, \tau)+\nabla\left[\mathbf{F}_{D}(\boldsymbol{R}) f(\boldsymbol{R}, \tau)\right]+\left[E_{L}(\boldsymbol{R})-E_{T}\right)\right] f(\boldsymbol{R}, \tau)
$$

where $\mathbf{F}_{D}(\boldsymbol{R})$ is the quantum force given by

$$
\mathbf{F}_{D}(\boldsymbol{R})=\nabla \ln \left|\Psi_{T}(\boldsymbol{R})\right|^{2}
$$

$E_{L}(\boldsymbol{R})$ is the local energy given by

$$
E_{L}(\boldsymbol{R})=\Psi_{T}(\boldsymbol{R})^{-1} \hat{H} \Psi_{T}(\boldsymbol{R})
$$

$D=1 / 2 m$ with $m$ being the mass of the atoms of the complex and $f(\boldsymbol{R}, \tau)=\Psi(\boldsymbol{R}, \tau) \Psi_{T}(\boldsymbol{R})$ is the distribution function.

The trial WF of the system is given by a product of purely nodeless exponential forms $[46]$

$$
\Psi_{T}(\boldsymbol{R})=\Psi_{T}\left(R_{1}, R_{2}, R_{3}\right)=\psi^{2 \mathrm{H}-\mathrm{He}}\left(R_{2}\right) \times \psi^{2 \mathrm{H}-\mathrm{He}}\left(R_{3}\right) \times \psi^{\mathrm{He}-\mathrm{He}}\left(R_{1}\right)
$$

where $f^{2 \mathrm{H}-\mathrm{He}}$ is the Jastrow correlation factor for the He-hydrogen interaction

$$
f^{2 \mathrm{H}-\mathrm{He}}\left(R_{i}\right)=-\left(\frac{p_{5}}{R_{i}^{5}}+\frac{p_{3}}{R_{i}^{3}}+\frac{p_{2}}{R_{i}^{2}}+p_{1} R_{i}+p_{0} \ln R_{i}\right)
$$

with $i=2$, 3 ; the Jastrow factor for the He-He pair has the same functional form.

The DMC procedure relies on the short-time approximation [45] whereby the Schrödinger equation is solved iteratively in the integral form through a relaxation process in imaginary time

$$
\Psi\left(\boldsymbol{R}^{\prime}, \tau_{k+1}\right)=\int G\left(\boldsymbol{R}^{\prime} \leftarrow \boldsymbol{R}, \tau\right) \Psi\left(\boldsymbol{R}, \tau_{k}\right) d \boldsymbol{R}
$$

where $\tau=\tau_{k+1}-\tau_{k}$ becomes now the discretized time step and the Green's function, given by

$$
G\left(\boldsymbol{R}^{\prime} \leftarrow \boldsymbol{R}, \tau\right)=\left\langle\boldsymbol{R}^{\prime}\left|\exp \left(-\tau\left(\hat{H}-E_{T}\right)\right)\right| \boldsymbol{R}\right\rangle
$$

can be interpreted as the "transition " probability to move to a new position $\boldsymbol{R}^{\prime}$ in the time step $\tau$. The projection operator in eq. (35) extracts the ground state WF $\Psi_{0}$ from an arbitrarily chosen initial state, written as a linear combination of the eigenfunctions $\Psi_{i}$ of $\hat{H}, \Psi_{0}=\sum_{i} c_{i} \Psi_{i}$, when $\tau \rightarrow \infty$

$$
\begin{array}{r}
\lim _{\tau \rightarrow \infty} \exp \left(-\tau\left(\hat{H}-E_{T}\right)\right) \sum_{i} c_{i} \Psi_{i}(\boldsymbol{R}) \Psi_{T}(\boldsymbol{R})=\lim _{\tau \rightarrow \infty} \sum_{i} \exp \left(-\tau\left(E_{i}-E_{T}\right)\right) c_{i} \Psi_{i}(\boldsymbol{R}) \Psi_{T}(\boldsymbol{R})= \\
\exp \left(-\tau\left(E_{0}-E_{T}\right)\right) c_{0} \Psi_{0}(\boldsymbol{R}) \Psi_{T}(\boldsymbol{R})
\end{array}
$$


The DMC ground state distribution function, $f_{0}=\Psi_{0} \Psi_{T}$, is reached by simulating the imaginary-time diffusion of replicas (walkers) of the system in the configurational space. The Green's function is generally unknown; in the importance sampling DMC framework $[45,47]$ the Green's function is splitted into two different contributions, according to the Trotter formula:

$$
\begin{aligned}
& G\left(\boldsymbol{R}^{\prime} \leftarrow \boldsymbol{R}, \tau\right) \simeq \tilde{G}\left(\boldsymbol{R}^{\prime} \leftarrow \boldsymbol{R}, \tau\right)=G_{d}\left(\boldsymbol{R}^{\prime} \leftarrow \boldsymbol{R}, \tau\right) G_{b}\left(\boldsymbol{R}^{\prime} \leftarrow \boldsymbol{R}, \tau\right) \\
& \quad=(4 \pi D \tau)^{-3 / 2} \exp \left[-\frac{\left[\boldsymbol{R}^{\prime}-\boldsymbol{R}-D \tau \mathbf{F}_{D}(\boldsymbol{R})\right]^{2}}{4 D \tau}\right] \exp \left\{\left[E_{T}-\frac{E_{L}(\boldsymbol{R})+E_{L}\left(\boldsymbol{R}^{\prime}\right)}{2}\right] \tau\right\}
\end{aligned}
$$

The $G_{b}=\exp \left\{\left[E_{T}-\frac{E_{L}(\boldsymbol{R})+E_{L}\left(\boldsymbol{R}^{\prime}\right)}{2}\right] \tau\right\}$ branching term can be considered as a rate term which rules the changes in the population of walkers [45]. From a "diffusional" point of view, this technique allows one to simulate the presence of "sources" and "sinks" in the imaginary time evolution of the process by replicating or "killing" the walkers. On average, walkers will die in regions where $\Psi_{T}>\Psi$ and give birth in regions where $\Psi_{T}<\Psi$. For the branching part, we follow the approach suggested by Assaraf et al. [48, 49]. At each $j$ th iteration we calculate the average branching factor for the $\mathrm{M}$ walkers

$$
W_{j}=\frac{1}{M} \sum_{i}^{M} b_{i, j}
$$

where the branching factors are calculated as in [50] i.e.

$$
b_{i, j}=\exp \left\{\left[E_{\text {Ref }}-\frac{E_{L}\left(\mathbf{R}_{i}\right)+E_{L}\left(\mathbf{R}_{i}^{\prime}\right)}{2}\right] \tau_{\text {eff }}\right\}
$$

and where $E_{L}\left(\mathbf{R}_{i}\right)$ and $E_{L}\left(\mathbf{R}_{i}^{\prime}\right)$ are the local energies before and after the move, $E_{R e f}$ is a constant value close to the eigenvalue and $\tau_{\text {eff }}$ is the effective timestep which depends on the acceptance/rejection ratio [47]. We then create a relative weight for each walker, given by

$$
\bar{\omega}_{i, j}=b_{i, j} / W_{j}
$$

The population of $\mathrm{M}$ walkers is reconfigured by dividing it in two subsets: there are $\mathrm{M}_{+}$ walkers with $\bar{\omega}_{i, j} \geq 1$ and $\mathrm{M}_{-}$walkers with $\bar{\omega}_{i, j}<1$. In order to maintain a constant population, a number of the $\mathrm{M}_{+}$walkers substitutes the same number of $\mathrm{M}_{-}$walkers. This number is given by [48]

$$
\operatorname{int}\left\{M_{\text {reconf }}+\eta\right)=\operatorname{int}\left\{\sum_{i+}^{M+}\left|\bar{\omega}_{i, j}-1\right|+\eta\right\}
$$


where $\eta$ is a uniform random number in $[0,1]$. The expectation value of an observable can therefore be written as the usual statistical average [46]:

$$
\langle\hat{O}\rangle=\frac{\int \Psi_{0}(\boldsymbol{R}) \hat{O} \Psi_{T}(\boldsymbol{R}) d \boldsymbol{R}}{\int \Psi_{0}(\boldsymbol{R}) \Psi_{T}(\boldsymbol{R}) d \boldsymbol{R}} \simeq \frac{1}{N_{b}} \sum_{k=0}^{N_{b}-1}\left[\left(\sum_{j=k \times M+1}^{(k+1) \times M} \omega_{j}\right)^{-1} \sum_{j=k \times M+1}^{(k+1) \times M} \omega_{j}\langle\hat{O}\rangle_{j}\right]
$$

where the $\langle\hat{O}\rangle_{j}$ is the ensemble average at the $j$-th iteration and the weight $\omega_{j}$ for each iteration is defined as a product over the last $L$ timesteps of the mean of the branching factors $W_{m}$ at the $m$-th iteration

$$
\omega_{j}=\prod_{m=j-L+1}^{j} W_{m}
$$

For the atomic dopants we have instead employed a DMC version [51-56] in which the number of walkers is changed during the simulation. Each walker is characterized by a cumulative weight; at the $j$-th iteration the weight $w_{i j}$ for the $i$-th walker is given by

$$
w_{i j}=\prod_{k=1}^{j} b_{i k}
$$

where $b_{i k}$ is the same as defined in Eq. 39. The only difference is in the reference energy $E_{R}$; it is now updated during the random walk, according to the following formula [50]

$$
E_{R}=E_{R}+\frac{\alpha}{\tau} \ln \frac{N\left(\tau_{j-1}\right)}{N\left(\tau_{j}\right)}
$$

where $\alpha$ is a control parameter (to be chosen small) and $N\left(\tau_{j-1}\right)$ and $N\left(\tau_{j}\right)$ represent the population of walkers in two successive steps of the random walk. Updating $E_{R}$ is a fundamental tool to minimize the fluctuations in the ensemble. Walkers are killed or replicated by using two parameters $w_{\min }$ and $w_{\max }$ :

- if $w_{i j}<w_{\min }$ the $i$-th walker is destroyed with probability $p_{-}=1-w_{i j}$ and retained with probability $p_{+}=w_{i j}$ and a weight equal to the average weight over the ensemble, $\bar{W}_{j}=\sum_{i}^{N} w_{i j}$

- if $w_{i j}>w_{\max }$ the $i$-th walker is replicated. The number of replicas is given by $N_{w}^{i j}=$ $\operatorname{int}\left(w_{i j}+\eta\right)$ where $\eta$ is uniform random number $\in[0,1]$. A new weight $w_{i j} / N_{w}^{i j}$ is associated at these $N_{w}^{i j}$ walkers;

- in the case $w_{\min } \leq w_{i j} \leq w_{\max }$ the $i$-th walker survives with no duplicates. 
The average over the ensemble at the $j$-th iteration becomes

$$
\langle\hat{O}\rangle_{j}=\frac{1}{W_{j}} \sum_{i=1}^{N} w_{i j} O_{i, j}
$$

where $W_{j}=\sum_{i}^{N} w_{i j}$. The final estimate of the observable $O$ is obtained by the usual definition of $N_{b}$ blocks of $M$ steps

$$
\langle\hat{O}\rangle=\frac{\int \Psi_{0}(\boldsymbol{R}) \hat{O} \Psi_{T}(\boldsymbol{R}) d \boldsymbol{R}}{\int \Psi_{0}(\boldsymbol{R}) \Psi_{T}(\boldsymbol{R}) d \boldsymbol{R}} \simeq \frac{1}{N_{b}} \sum_{k=0}^{N_{b}-1}\left[\frac{1}{M} \sum_{j=k \times M+1}^{(k+1) \times M}\langle\hat{O}\rangle_{j}\right] .
$$

\section{BOUND STATE CALCULATIONS}

\section{A. The atom-atom potentials}

In the study of very small weakly bound VdW systems, the relevant potential energy surface (PES) employed to assess the presence of possible bound states of that complex is usually constructed as sums of the $2 \mathrm{~B}$ potentials between the interacting partners, i.e. disregarding the effects from the $3 \mathrm{~B}$ forces usually taken to be nearly negligible in such special systems

$$
V_{T O T}\left(R_{1}, R_{2}, R_{3}\right)=V_{H e-H e}\left(R_{1}\right)+\sum_{i=1}^{2} V_{H e-H_{i}}\left(R_{i+1}\right)+O\left(V_{3 B}\right) .
$$

In the case of the atomic impurity $\mathrm{H}$ and its isotopic variants we employ in the present study different atom-atom potentials with respect to the other calculations reported in literature $[8,12]$. The He-He interaction was selected among the many existing proposals (which we extensively reviewed a while ago [57]), where we found that one of the potentials proposed by Aziz and coworkers [58] was indeed able to support the single bound state of the dimer for $J=0$. The potential selected will be labelled the HFDID potential from [58]. We then adopted the model potential of Ref. [59] to describe the He-H interaction because of its accuracy in describing the $2 \mathrm{~B}$ properties of the system. Our comparison with Lin's potential choices showed us that the present $2 \mathrm{~B}$ potentials are very similar to those employed by him and therefore we carried out our calculations by using only our present selection, expecting it to yield comparable results.

The interaction between any of the He atoms and the $\mathrm{H}_{2}$ molecule was taken instead from Ref. [17], the same anisotropic interaction which we have used in the earlier calculations on $\mathrm{H}_{2}-\mathrm{He}[16]$, and which has been obtained from accurate ab-initio calculations. 
The potential curves for all the atom-atom subsystems employed in the present work are shown in Fig. 1. In that figure we also give indications on the anisotropic strength of the full $\mathrm{H}_{2}$-He surface [17] in comparison with its isotropic component, an item of interest for the present work.

The following comments could be made from a perusal of the data in the figure:

1. the two potential curves associated with the $\mathrm{H}_{2}$-He fully anisotropic interaction (labelled as BMP) indicate clearly that the repulsive regions are very similar in both orientations $(\theta=0$ or $\pi / 2)$ and also have similar long-range behavior. In other words, the $\mathrm{H}_{2}$-He system exhibits a largely isotropic interaction potential;

2. if we consider the spherical component of the $\mathrm{H}_{2}$-He surface (labelled $\mathrm{V}_{0}$ in the figure) we see that it follows very closely both orientations' behavior, with only minor differences around the well depth value;

3. the He-H interaction is the weakest one for the three subsystems considered here, but it shows the same spatial extention than the $\mathrm{H}_{2}$-He interaction;

4. the He-He interaction, known to be a very weak interaction potential, is seen here (labelled as HFDID) to be only slightly weaker than the $\mathrm{H}_{2}$-He interaction potential while it is stronger than the He-H interaction. The He-He potential well depth is then localized at smaller atom-atom distances with respect to the potentials of the helium atoms with both hydrogen moieties.

\section{B. The atomic partners: the hydrogen and its isotopic variants}

1. The trimers: $\mathrm{H}(\mathrm{He})_{2}, \mathrm{D}(\mathrm{He})_{2}$ and $\mathrm{T}(\mathrm{He})_{2}$

The DGF method has been applied for the cases of the three isotopic variants, using 711 Gaussian functions along the He-H coordinate (with the outermost one located at $10000 \mathrm{a}_{0}$ ) while 103 functions (up to $360 \mathrm{a}_{0}$ ) were sufficient to fully describe the more compact He-He interaction domain. The resulting number of basis functions was 626,152. For the Faddeev calculation, we have employed up to $n=8$ adiabatic terms in the expansion of the WF from Eq. (21). Orbital angular momenta $\ell_{x}, \ell_{y} \leq 4$ have been considered in Eq. (22) and the maximum hypermomentum values $\mathcal{K}_{\max }$ used for each of the components are given in 
Table I. Finally, the coupled set of radial equations given in Eq.(24) has been solved using a Runge-Kutta procedure up to a maximum value of the hyperradius $\rho$ of about $475 a_{0}$. The DMC calculation employed 2000 walkers propagated in 40 blocks composed by 60,000 timesteps of 300 hartree $^{-1}$.

As we can see from the parameters employed in the present calculations, the analysis of the possible bound states associated with the $\mathrm{H}$ atom can become quite demanding in terms of computational effort. In particular, the DGF approach requires a rather large distribution of basis functions in order to map the extended length of those "bonds" and ensure the converged control on the location of possible bound states. The corresponding eigenproblem would be really hard, if not impossible, to solve with standard diagonalization techniques, while only a moderate computational effort is required by the present implementation of the JD procedure within the DGF scheme.

Although no bound states were found for either $\mathrm{H}(\mathrm{He})_{2}$ or $\mathrm{D}(\mathrm{He})_{2}$, it is instructive to look at the results reported by Fig. 2 for the computed pair distribution functions along the He-D distance obtained by means of the DGF approach.

The computations, carried out with the expansion details given above, show the extensions of the pair distribution functions along the He-D bonds. Such functions are plotted for each converged variational calculation where the location of the outermost Gaussian function is moved to increasingly larger radial values.

The results indicate that, in the absence of the formation of a truly bound complex, to employ increasingly larger "boxes" whithin which to search for bound states forces the distributions to map out the entire available space since we are essentially describing dissociative states near the energy threshold of the $\mathrm{He}_{2}+\mathrm{D}$ partners.

On the other hand, when the same procedure is carried out for the $\mathrm{T}(\mathrm{He})_{2}$ system, we found that beyond certain distances the pair distribution does not change anymore and both of them clearly converge to the quantum distributions of a bound trimer. The data reported by Fig. 3, in fact, refer to the final distributions and show the much more compact nature of these quantities when the complex is bound. In other words, even when the physical "box" chosen to describe the He-H interaction range is extended to $10000 \mathrm{a}_{0}$, the formation of a bound state is signaled by the more rapid convergence of the radial pair distribution functions and the essentially negligible density values beyond about $100 \mathrm{a}_{0}$.

The comparison between the DGF, Faddeev and DMC (mixed estimator) pair distri- 
butions reported by the same Fig. 3 now reveals an excellent agreement between DMC and Faddeev methods, while the DGF apparently deviates slightly from the overall picture. However, we see that all three methods yield probability density functions which display peaks at basically the same values of the He-He and He-T pair distances. The only discrepancy seems to be about the total extension of the distributions, with the DMC and Faddeev results extending to slightly larger distances than the DGF data, the difference being more marked for the He-T length. As a consequence, predictions for the mean values of the atomatom distances, estimated from the 1D distribution reported in Fig. 3 reveal larger values for the Faddeev and DMC results (both yielding $27.7 \mathrm{a}_{0}$ and $34.7 \mathrm{a}_{0}$, for the $\mathrm{He}-\mathrm{He}$ and He-T distances respectively) than for the corresponding DGF estimates (22.6 $\mathrm{a}_{0}$ and 26.5 $\mathrm{a}_{0}$ ). However, it should be noted that the He-T atom-atom distribution now extends over 100 a.u., meaning that the mean radial values differ by $5 \%$ (for the $\mathrm{He}-\mathrm{He}$ ) and by $8 \%$ (for the $\mathrm{He}-\mathrm{T}$ ) with respect to the total spatial extension: an acceptable discrepancy given the special nature of this complex and the very small values of its binding energy.

Furthermore, the overall geometry given by both methods is not far from an equidistant location of each He atom with respect to the other helium partner and to the $\mathrm{H}$ atom. This feature further confirms the extreme weakness of the He-He interaction which, although able to support one $2 \mathrm{~B}$ bound state, yields a bond distance within the $\mathrm{He}_{2}-\mathrm{H}$ complex perfectly comparable to the corresponding separation between the $\mathrm{T}$ and He atoms, which in fact are not capable of supporting a separate $2 \mathrm{~B}$ bound system.

As for the energy value of the bound state, we saw that the DGF calculations which map radial distances beyond about $300 \mathrm{a}_{0}$ essentially make no changes on the final eigenvalue. In other words, the size of the integration box has essentially reached its limit, beyond which the corresponding bound state WF has all but vanished. The extrapolated values at zero badness for each chosen step $\Delta$ are reported in the second column of Table II for decreasing values of the step. By fitting these energies as a function of $\Delta$ and by extrapolating to the $\delta$-function limit $(\Delta=0)$, as discussed in detail in the previous section, we obtain the final value of $-0.01407 \mathrm{~cm}^{-1}$ for the only bound state of the $\mathrm{T}(\mathrm{He})_{2}$ at $J=0$. The Faddeev result is here $-0.01409 \mathrm{~cm}^{-1}$, in extremely good agreement with the DGF prediction. A test of the convergence in terms of the number of radial expansion coefficients $f_{n}(\rho)$ from Eq. (21) is shown in Table III. Finally, DMC calculations produce a more strongly bound $\mathrm{T}(\mathrm{He})_{2}$, with an energy of $-0.01898(20) \mathrm{cm}^{-1}$. Given the smallness of the energy involved, however, it is 
not entirely surprising to find that an energy "mismatch " between the methods of the order of $10^{-3} \mathrm{~cm}^{-1}$ looks like a sizeable difference, although it really corresponds to a rather small difference, in absolute terms, between energies computed with different procedures.

To further give us some pictorial representation of this very special state, we report in Fig. 4 the contour plot and a three-dimensional perspective along the He-He and $\mathrm{T}(\mathrm{He})_{2}$ distances for the probability density function obtained via the Faddeev approach of Eq. (28). The radial density function displays a similar behaviour for both coordinates, thus supporting an overall structure for the complex with the $\mathrm{H}$ atom located from the center of mass of the $\mathrm{He}_{2}$ unit at a similar distance to that for the He-He bond.

In Fig. 5 we further show an analysis of the relative weights of the most significant triangle configurations that describe the bound trimer. The results from Fig. 5 further confirm, albeit more quantitatively, the spatial features of this bound state found by our calculations: the linear configurations of this very floppy system are substantial in number (about $20 \%$ ) and describe an "outer" hydrogen atom attached to the helium dimer (see mid and bottom panels of that figure). On the other hand, the scalene structures (i.e. the distorted isosceles triangles) give the dominant contributions to the description of the system: a very floppy He dimer with an outer T atom hovering around it at distances of the same magnitude as the separation between the He atoms.

Finally, a further search for possible states with Efimov character leads to the conclusion that, given our current knowledge of $2 \mathrm{~B}$ forces, no Efimov state exists for the $\mathrm{T}(\mathrm{He})_{2}$ complex. Bound state calculations performed by means of the DGF method when the total strength of the potential was artificially increased reveal that the energy of the trimer always remain below the corresponding value of the $\mathrm{He}_{2}$ bound state.

\section{Strengthening the "cage" effects: $\mathrm{H}(\mathrm{He})_{3}$ and $\mathrm{D}(\mathrm{He})_{3}$}

In the previous Section we have discussed how the change in the mass of the impurity reflects on the existence of a bound state within a two-He atomic 'cage'. Doubling the impurity mass from $\mathrm{H}$ to $\mathrm{D}$ turned out not to stabilize the trimer for a bound state to appear: the hydrogen-helium interaction is so weak that we needed to use the heaviest hydrogen isotope to find a bound state for the trimer. In the present Section we additionally analyze the effect of slightly enlarging the He-atom cage on the system's bound spectrum 
and, again, we start with the lightest $\mathrm{H}$ isotope. For the four-body system we only carry out DMC calculations, reassured by its good agreement with DGF and Faddeev methods already employed for the trimers of the previous Section. For the $\mathrm{H}(\mathrm{He})_{3}$ system the DMC calculations find a state with energy $-0.087(2) \mathrm{cm}^{-1}$. Such a state is essentially isoenergetic with respect to the $\mathrm{He}_{3}$ system $\left(-0.0877(4) \mathrm{cm}^{-1}\right.$, calculated by us) which, hence, does not seem to be capable to bind the very light impurity. On the other hand, the tetramer $\mathrm{D}(\mathrm{He})_{3}$ shows a more marked bound state with energy $-0.13166(57) \mathrm{cm}^{-1}$. Our zero of energy refers, as usual, to the full fragmentation, i.e. to the atomic components; with respect to the deuterium loss the binding energy is instead $4 \times 10^{-2} \mathrm{~cm}^{-1}$, which is around $57 \mathrm{mK}$. This extremely weakly bound system is, as expected, highly delocalized in space, as can be seen from Fig. 6, where we report the 1D radial and angular distributions which characterize the $\mathrm{D}(\mathrm{He})_{3}$ spatial features. We notice there that the $\mathrm{D}$ species, though bound to the three He atoms, is clearly 'outside' the helium cluster and quite far away, spanning an impressive radial box of the order of the $\mu \mathrm{m}$. Such a microscopic extension reflects on the distribution function obtained for the He-D-He angular variable: the D atom is so far away that the relative angle is always close to 0 . The angular distribution as a function of the D-He-He angle is thus flat and shapeless over the entire definition domain, further indication of the very large spatial delocalization of the $\mathrm{D}$ impurity.

Since we had already found the $\mathrm{T}$ isotope to be bound to two He atoms, we did not think to be of special interest in this work to further confirm it in the case of three He atoms. We shall, in fact, present and discuss the T-solvation in larger He clusters in a separate study [60].

\section{The molecular partner: $\mathrm{H}_{2}$ and $(2 \mathrm{H})$}

Because of the very marked weakness of the present system, and also in order to test the structural importance of including the full anisotropy of the $\mathrm{He}_{-} \mathrm{H}_{2}$ "molecular " interaction, we carried out calculations using either the full He- $\mathrm{H}_{2}$ PES of Ref. [17] or its spherically averaged potential also shown by Fig. 1. The DMC calculations were preceded by variational, VMC, calculations to optimize the trial WF $\Psi_{T}$ needed in the importance sampling. The DMC procedure involved 4000 walkers, time steps of 100 hartree $^{-1}$, 100 blocks and 6000 iterations per block. Such data apply to "atom-like" calculations pertaining to the 
$(2 \mathrm{H})(\mathrm{He})_{2}$ system, while the "molecular "calculations for the $\mathrm{H}_{2}(\mathrm{He})_{2}$ system employed, to reach convergence, 1500 walkers, time steps of 200 hartree $^{-1}, 20$ blocks and 6000 iterations per block [9]. The DGF calculations were applied to the $(2 \mathrm{H})(\mathrm{He})_{2}$ case and employed a total of 206 distributed Gaussians functions over a radial range of $200 \AA$ for the He-He bond and for the (2H)-He bonds: only "atomic-like" calculations were carried out in this case. The final badness value was extrapolated to zero and the initial width of the Gaussian functions was chosen to be 0.6 bohr, also extrapolated to near-zero width in the final calculations (see previous Section II for further details).

The numerical results are collected in Table IV. Two different He-He potentials were used: the HFDID from Ref. [58], which we discussed before, and the TT potential from Ref. [61] that has been often suggested also as a possible potential. The results from the Table clearly indicate the good accord in achieved between the two methods. Furthermore, we see that a fully anisotropic $\mathrm{He}-\mathrm{H}_{2}$ potential and its spherical modelling yield essentially very similar binding energies: the differences are of the order of about $0.01 \mathrm{~cm}^{-1}$. Finally, the choice of two different He-He potentials, of which the TT one does not produce a bound He dimer, also appears to cause fairly small differences. The more realistic HFDID potential (Fig. 1) produces a smaller binding energy value, but only by about 0.013 (DMC) or 0.016 (DGF) $\mathrm{cm}^{-1}$. Such calculation, therefore, can give us some feeling about the possible "sensitivity" of our results on the chosen $2 \mathrm{~B}$ potentials.

In conclusion, one sees that the present four-particle system is indeed very weakly bound and it can be just as accurately described as a three-particle species containing a spherical $(2 \mathrm{H})$ partner. It is usually understood that the calculation of the radial distributions between partners, especially between very weakly bound species as in the present instance, provides a very sensitive test on the comparable qualities of different computational procedures. It is therefore very useful to further evaluate radial densities along both the He-He and the He- $(2 \mathrm{H})$ bonds in order to compare the findings of the DGF and DMC schemes discussed earlier.

The results are reported by the two panels of Fig. 7, the top one being for the HeHe distances and the bottom one for the He- $(2 \mathrm{H})$ distances. The solid lines describe the radial densities associated with the DMC results (obtained by the mixed estimator), while the dashes report the results obtained using the DGF method described in the previous Section. It is once again remarkable, and rather reassuring, to see that both methods yield 
here essentially the same distributions along the two distances. Furthermore, their maxima appear roughly at the same radial value of about 10 bohr, thus indicating the preferential collocation of this very floppy, quantum system as an equilateral triangular structure: we indeed obtained the same result by using the full anisotropic PES in our earlier calculations [9]. One further indicator of the highly quantum nature of this system is given by the estimates of zero-point energies which can be obtained from the binding energies computed here: the well depth of the two $2 \mathrm{~B}$ potentials are around $10 \mathrm{~cm}^{-1}$ and $7 \mathrm{~cm}^{-1}$ for He-He, while we obtain binding energies which are around a fourth of $\mathrm{a} \mathrm{cm}^{-1}$. This feature therefore suggests that more than $95 \%$ of the total energy available is given as a classically excluded region for the weakly bound, highly delocalized quantum system. This finding is also very similar to the well-known behavior of the He dimer [62].

\section{DISCUSSION OF RESULTS AND CONCLUSIONS}

As already mentioned in the Introduction, the study of ultra-weak interaction forces which might bound small atomic clusters pushes to their computational limits all the standard methods which are commonly employed for the calculations of bound states. This is chiefly due to the unusual extension of the spatial domain of definition for the bound WFs, as can be inferred by the features of pair distribution functions and by the values of the variational parameters required for convergence shown in the previous Section. Additionally, to have to deal with binding energies of the order of the $\mathrm{mK}$ (or fraction of $\mathrm{mK}$ ) constitutes a significant challenge for getting converged results and, as illustrated in the case of the $\mathrm{T}(\mathrm{He})_{2}$ system, small discrepancies obtained when using different, independent methods are not surprising, especially when the characterization of the system in terms of its spatial attributes shows a substantial agreement between different approaches.

The DGF representation of the Hamiltonian was introduced ten years ago precisely with the aim of providing a procedure more suited to the characterization of weakly bound systems and the Jacobi-Davidson scheme has enabled us to increase by more of one order of magnitude the number of basis Gaussian functions employed in the calculation while reducing the computational time and the memory required by the method. The DMC and Faddeev procedures are usually employed in the study of more conventional, nearly chemically bound systems, while the interaction of one hydrogen atom with two helium moieties 
constitutes for them a very challenging case, due to the nature of the potentials involved. It is well known that one He atom never binds to a single hydrogen atom: the interaction potential, reported in Fig. 1, is too weak to support a bound state between the two very light particles. The replacement of $\mathrm{H}$ with its heaviest isotopes, deuterium and tritium, which represents the most dramatic isotopic mass effect for all known elements, does not cause the appearance of any bound state.

On the other hand, the $\mathrm{H}_{2}$-He system does present a bound state: in the spherical approximation of the interaction the molecular hydrogen is really treated as a deuterium atom so that the different potential for the $\mathrm{H}_{2}$-He system has the effect of "tuning" the known interaction potential on the He-D dimer: indeed the $\mathrm{He}-\mathrm{H}_{2}$ potential, though comparable to the latter in terms of spatial extension and position of the well, changes enough to be able to support a bound state.

A further way to act on the system has been provided by a small increase of the solvating 'shell' of the He atoms. Even though the H-He dimer is not bound, the addition of a second He to the H-He dimer is seen to create the conditions for a Halo state to appear, i.e., for a bound trimer state with unbound diatomic subsystems.

In sum, the above studies have allowed us to analyze in detail the possible existence of bound states (with $J=0$ ) for all the isotopic variants of an hydrogen atom interacting with the helium dimer in its bosonic form. The intermolecular forces, constructed as a sum of $2 \mathrm{~B}$ interactions, are known to be very weak in such systems and therefore it was not at all clear in advance whether or not such bound complex structures exist within the current quality of $2 \mathrm{~B}$ forces available.

Our calculations have employed a variational expansion over a symmetry-adapted, nonorthogonal basis of Gaussian functions, the DGF expansion [21, 22], the Faddeev equations and the DMC treatment: we have carefully controlled the detailed numerical convergence of all the parameters which appear within all three methods.

All three approaches used the same modelling of the 3B interaction as described earlier and provided in the end remarkable agreement with each other, finding only one bound state for the $\mathrm{T}(\mathrm{He})_{2}$ system and the same energy eigenvalue for such state. These systems further showed very similar atom-atom density functions and agreed on indicating that the $\mathrm{T}(\mathrm{He})_{2}$ bound trimer should not support any excited state with Efimov character.

In conclusion, we have shown through different examples how it is possible to achieve 
accurate and reliable evaluations of the binding energies and of the bound state features for the smaller (trimers and tetramers) clusters that interact via ultraweak forces, exhibiting very unusual spatial features and properties with respect to more conventional, and more strongly bound, "chemical" systems.

\section{CAPTIONS}

- Fig 1: 2B potential term contributions employed for the $\mathrm{H}-\mathrm{He}_{2}$ and the (2H)-He systems discussed in this work. The He-He potential is from Ref. [58], the $\mathrm{H}_{2}-\mathrm{He}$ potential curves are from Ref. [17], while the H-He potential is from Ref. [59]. See main text for further details.

- Fig 2: Computed pair distributions along the ${ }^{4} \mathrm{He}-\mathrm{D}$ bound distance obtained with the DGF method as a function of the location of the outermost Gaussian function.

- Fig 3: Computed pair distribution $\mathcal{D}_{D G F}^{(0)}$ and $\mathcal{D}_{\text {Faddeev }}^{(i)}$ functions (see Eqs. (27) and (6)) as calculated with the DGF (solid line), Faddeev (dashed line with filled circles) and DMC (dashed line with stars) approaches along the He-He (left panel) and He-T (right panel) bonds within the bound triatomic complex. Distances are measured in $a_{0}$. See text for further details.

- Fig 4: Three dimensional perspective and contour plots for the probability density in terms of the He-He and $\mathrm{T}_{-} \mathrm{He}_{2}$ distances for the $\mathrm{T}(\mathrm{He})_{2}$ bound state obtained by means of the Faddeev calculation. See Eq. (28).

- Fig 5: Computed pseudo-weights for all triangular configurations of the $\mathrm{T}(\mathrm{He})_{2}$ system (top panel). The middle panel shows the triangular configurations centered on either ${ }^{4} \mathrm{He}$ atoms, while the bottom panel shows those centered on the $\mathrm{T}$ atom.

- Fig 6: 1D radial probability functions and, in the inset, the angular distribution for the $\mathrm{D}-{ }^{4} \mathrm{He}_{3}$ system.

- Fig 7: Computed radial densities using both the DMC (solid lines) and the DGF (dashes) methods. The upper panel refers to the He-He distance while the lower panel reports the $(2 \mathrm{H})$-He distance. See text for details. 


\section{Acknowledgments}

The financial support of the University of Rome Research Committee, of the CASPUR Supercomputing Center and of the PRIN 2006 Research Programme is gratefully acknowledged. This work has been supported by the Spanish MICINN under projects FIS2007-62006 and FIS2008-01301. We also acknowledge the support of the INTAS collaboration and of the Italy-Spain Integrated Action Programme. 
[1] S. Grebenev, J. P. Toennies, and A. F. Vilesov, Science 279, 2083 (1998).

[2] W. Schollkopf and J. P. Toennies, Science 266, 1345 (1994).

[3] K. K. Lehmann and G. Scoles, Science 279, 2065 (1998).

[4] J. P. Toennies and A. F. Vilesov, Angew. Chem. Int. Ed. 43, 2622 (2004).

[5] D. DeMille, Phys. Rev. Lett. 88, 067901 (2002).

[6] J. M. Doyle and B. Friedrich, Nature 401, 749 (1999).

[7] S. Orlandini, I. Baccarelli, and F. A. Gianturco, Mol. Phys. 106, 573 (2008).

[8] Y. D. Liu and P.-N. Roy, J. Chem. Phys. 121, 6282 (2004).

[9] E. Coccia, E. Bodo, and F. A. Gianturco, J. Chem. Phys. 130, 094906 (2009).

[10] R. A. Aziz and M. J. Slaman, J. Chem. Phys. 94, 8047 (1991).

[11] W. Meyer and L. Frommhold, Theor. Chim. Acta 88, 201 (1994).

[12] Y. Li and C. D. Lin, J. Phys. B 32, 4877 (1999).

[13] R. N. Barnett and K. B. Whaley, J. Chem. Phys. 96, 2953 (1992).

[14] M. C. Gordillo, Phys. Rev. B 60, 6790 (1999).

[15] A. Kalinin, O. Kornilov, L. Y. Rusin, and J. P. Toennies, J. Chem. Phys. 121, 625 (2004).

[16] F. A. Gianturco, T. Gonzalez-Lezana, G. Delgado-Barrio, and P. Villarreal, J. Chem. Phys. 122, $084308(2005)$.

[17] A. I. Boothroyd, P. G. Martin, and M. R. Peterson, J. Chem. Phys. 119, 3187 (2003).

[18] S. Orlandini, I. Baccarelli, and F. A. Gianturco, Comp. Phys. Comm. 180, 384 (2008).

[19] T. Gonzalez-Lezana, J. Rubayo-Soneira, S. Miret-Artes, F. A. Gianturco, G. Delgado-Barrio, and P. Villarreal, Phys. Rev. Lett. 82, 1648 (1999).

[20] T. González-Lezana, J. Rubayo-Soneira, S. Miret-Artés, F. A. Gianturco, G. Delgado-Barrio, and P. Villarreal, J. Chem. Phys. 110, 9000 (1999).

[21] S. Orlandini, I. Baccarelli, and F. A. Gianturco, J. Chem. Phys. 125, 234307 (2006).

[22] I. Baccarelli, F. A. Gianturco, T. González-Lezana, G. Delgado-Barrio, S. Miret-Artés, and P. Villarreal, Phys. Rep. 452, 1 (2007).

[23] E. Nielsen, D. V. Fedorov, A. S. Jensen, and E. Garrido, Phys. Rep. 347, 373 (2001).

[24] I. Baccarelli, G. Delgado-Barrio, F. A. Gianturco, T. González-Lezana, S. Miret-Artés, and P. Villarreal, Europhys. Lett. 50, 567 (2000). 
[25] I. P. Hamilton and J. C. Light, J. Chem. Phys. 84, 306 (1986).

[26] I. Baccarelli, F. A. Gianturco, T. González-Lezana, G. Delgado-Barrio, S. Miret-Artés, and P. Villarreal, J. Chem. Phys. 122, 084313 (2005).

[27] S. Orlandini, I. Baccarelli, and F. A. Gianturco, Molecular Physics 106, 573 (2008).

[28] G. L. G. Sleijpen and H. A. V. der Vorst, SIAM J. Mat. Anal. and Appl. 17, 401 (1996).

[29] A. Ern and J. Guermond, Theory and practice of finite elements (Springer Verlag, New York, 2004).

[30] D. R. Fokkema, G. L. G. Sleijpen, and H. A. V. der Vorst, SIAM J. Sci. Comput. 20, 94 (1998).

[31] E. Garrido, D. V. Fedorov and A. S. Jensen, Phys. Rev. C 55, 1327 (1997).

[32] E. Garrido and E. Moya de Guerra, Nucl. Phys. A 650, 387 (1999).

[33] E. Garrido, D. V. Fedorov and A. S. Jensen, Europhys. Lett. 43, 386 (1998).

[34] E. Garrido, D. V. Fedorov and A. S. Jensen, Nucl. Phys. A 700, 117 (2002).

[35] C. Romero-Redondo, E. Garrido, D. V. Fedorov and A. S. Jensen, Phys. Lett. B 32, 660 (2008).

[36] E. Garrido, D. V. Fedorov and A. S. Jensen, Nucl. Phys. A 733, 85 (2004).

[37] E. Garrido, D. V. Fedorov and A. S. Jensen, Eur. Phys. J. A 25, 365 (2005).

[38] R. Álvarez-Rodríguez, E. Garrido, D. V. Fedorov, A. S. Jensen and H. O. U. Fynbo, Eur. Phys. J. A 31, 303 (2007).

[39] R. de Diego, E. Garrido, D. V. Fedorov and A. S. Jensen, Nucl. Phys. A 786, 71 (2007).

[40] C. Romero-Redondo, E. Garrido, D. V. Fedorov and A. S. Jensen, Phys. Rev. C 77, 054313 (2008).

[41] V. Efimov, Phys. Lett. B 33, 563 (1970).

[42] V. Efimov, Sov. J. Nucl. Phys. 12, 589 (1971).

[43] V. Efimov, Nucl. Phys. A 210, 157 (1973).

[44] D. V. Fedorov and A. S. Jensen, Phys. Rev. Lett. 71, 4103 (1993).

[45] B. L. Hammond, W. A. Lester, Jr., and P. J. Reynolds, Monte Carlo Methods in Ab-Initio Quantum Chemistry (World Scientific, Singapore, 1994).

[46] E. Bodo, E. Coccia, D. Lopez-Duran, and F. A. Gianturco, Phys. Scripta 76, C104 (2007).

[47] P. J. Reynolds, D. M. Ceperley, B. J. Alder, and W. A. Lester, Jr., J. Chem. Phys. 77, 5593 (1982). 
[48] R. Assaraf, M. Caffarel, and A. Khelif, Phys. Rev. E 61, 4566 (2000).

[49] I. Bosá and S. M. Rothstein, J. Chem. Phys. 121, 4486 (2004).

[50] A. Viel, M. V. Patel, P. Niyaz, and K. B. Whaley, Comp. Phys. Comm. 145, 24 (2002).

[51] D. Blume, M. Lewerenz, F. Huisken, and M. Kaloudis, J. Chem. Phys. 105, 8666 (1996).

[52] F. Paesani, F. A. Gianturco, M. Lewerenz, and J. P. Toennies, J. Chem. Phys. 112, 2239 (2000).

[53] F. Paesani and F. A. Gianturco, J. Chem. Phys. 116, 10170 (2002).

[54] F. Paesani and F. A. Gianturco, J. Chem. Phys. 117, 709 (2002).

[55] C. Di Paola, F. Sebastianelli, E. Bodo, I. Baccarelli, F. A. Gianturco, and M. Yurtsever, J. Chem. Theory and Comput. 1, 1045 (2005).

[56] C. Di Paola, E. Bodo, and F. A. Gianturco, Eur. Phys. J. D 40, 377 (2006).

[57] C. Di Paola, F. Gianturco, G. Delgado-Barrio, S. Miret-Artés, and P. Villarreal, Czech. Chem. Commun. 68, 1 (2003).

[58] R. A. Aziz and M. J. Slaman, J. Chem. Phys. 94, 8047 (1991).

[59] U. Kleinekathofer, K. T. Tang, J. P. Toennies, and C. L. Yiu, Chem. Phys. Lett. 249, 257 (1996).

[60] E. Coccia and F. A. Gianturco, J. Phys. Chem. A submitted (2009).

[61] K. T. Tang and J. P. Toennies, J. Chem. Phys. 118, 4976 (2003).

[62] J. Q. W. Lo and B. D. Shizgal, J. Phys. B: At. Mol. Opt. Phys. 41, 185103 (2008). 


\begin{tabular}{|ccc|ccc|}
\hline \multicolumn{3}{|c|}{$i=1$} & \multicolumn{3}{c|}{$i=2,3$} \\
\hline$\ell_{x}$ & $\ell_{y}$ & $\mathcal{K}_{\max }$ & $\ell_{x}$ & $\ell_{y}$ & $\mathcal{K}_{\max }$ \\
\hline 0 & 0 & 1700 & 0 & 0 & 1700 \\
2 & 2 & 254 & 1 & 1 & 202 \\
4 & 4 & 168 & 2 & 2 & 204 \\
& & & 3 & 3 & 166 \\
& & & 4 & 168 \\
\hline
\end{tabular}

TABLE I: Components included in the Faddeev calculation for the expansion in Eq. (22). $\mathcal{K}_{\max }$ gives the maximum value of the hypermomentum used in the calculations. For $i=1$ the $\mathbf{x}_{i}$ Jacobi coordinate goes between the two He atoms, while for $i=2,3$ it goes between the He and the $\mathrm{H}$ atoms.

\begin{tabular}{|c|c|c|c|}
\hline $\mathrm{He}_{2} \mathrm{~T}$ & $\mathrm{E}_{0}\left(\mathrm{~cm}^{-1}\right)$ & DGFs & basis functions \\
\hline \hline$\Delta=1.2$ & -0.00096 & 144 & 556,851 \\
$\Delta=1.1$ & -0.00330 & 157 & 731,077 \\
$\Delta=1.0$ & -0.00565 & 172 & 949,889 \\
$\Delta=0.9$ & -0.00745 & 193 & $1,334,099$ \\
$\Delta=0.8$ & -0.00881 & 217 & $1,890,668$ \\
\hline \hline$\Delta=0$ & -0.01407 & & \\
\hline
\end{tabular}

TABLE II: Details of the extrapolation procedure to the $\Delta=0$ case in the DGF calculation for the ${ }^{4} \mathrm{He}_{2} \mathrm{~T}$. We have used DGFs with single step from $3.0 \mathrm{a}_{0}$ to $100.0 \mathrm{a}_{0}$ and with double step from $100.0 \mathrm{a}_{0}$ to $300.0 \mathrm{a}_{0}$. See text for further details.

\begin{tabular}{|c|cccccccc|}
\hline & $n=1$ & $n=2$ & $n=3$ & $n=4$ & $n=5$ & $n=6$ & $n=7$ & $n=8$ \\
\hline$E\left(\mathrm{~cm}^{-1}\right)$ & -0.01086 & -0.01138 & -0.01283 & -0.01352 & -0.01362 & -0.01398 & -0.01405 & -0.01409 \\
\hline
\end{tabular}

TABLE III: Convergence test with respect to the number $n$ of adiabatic terms in the expansion of Eq. (21). 


\begin{tabular}{|c|c|c|c|c|}
\hline \hline & \multicolumn{2}{|c|}{ DMC } & \multicolumn{2}{c|}{ DGF } \\
\hline $\mathrm{H}_{2}-\mathrm{He}_{2}$ & - & $-0.248(3)^{b}$ & - & - \\
$(2 \mathrm{H})-\mathrm{He}_{2}$ & $-0.272(2)^{a}$ & $-0.259(2)^{b}$ & $-0.272^{a}$ & $0.256^{b}$ \\
\hline \hline
\end{tabular}

TABLE IV: Computed total energies for $\mathrm{H}_{2}-\mathrm{He}_{2}$ and $(2 \mathrm{H})-\mathrm{He}_{2}$ using both the DMC and the DGF methods. ${ }^{a}$ HFDID He-He potential from Ref. [58]; ${ }^{b}$ TT He-He potential from Ref. [61]; the energy value of $-0.248(3) \mathrm{cm}^{-1}$ from Ref. [9]. See text for details. 


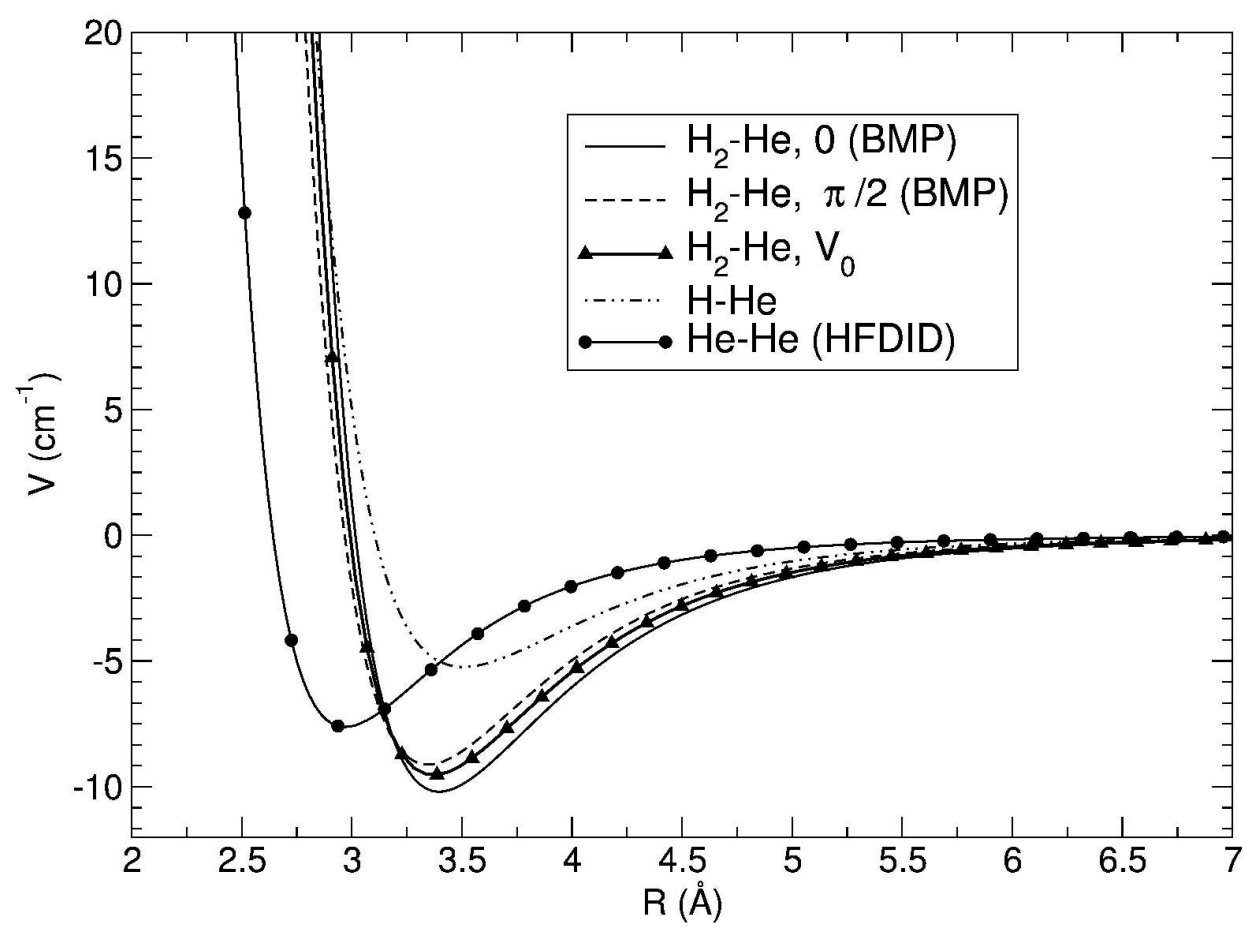

$279 \times 215 \mathrm{~mm}(600 \times 600 \mathrm{DPI})$ 


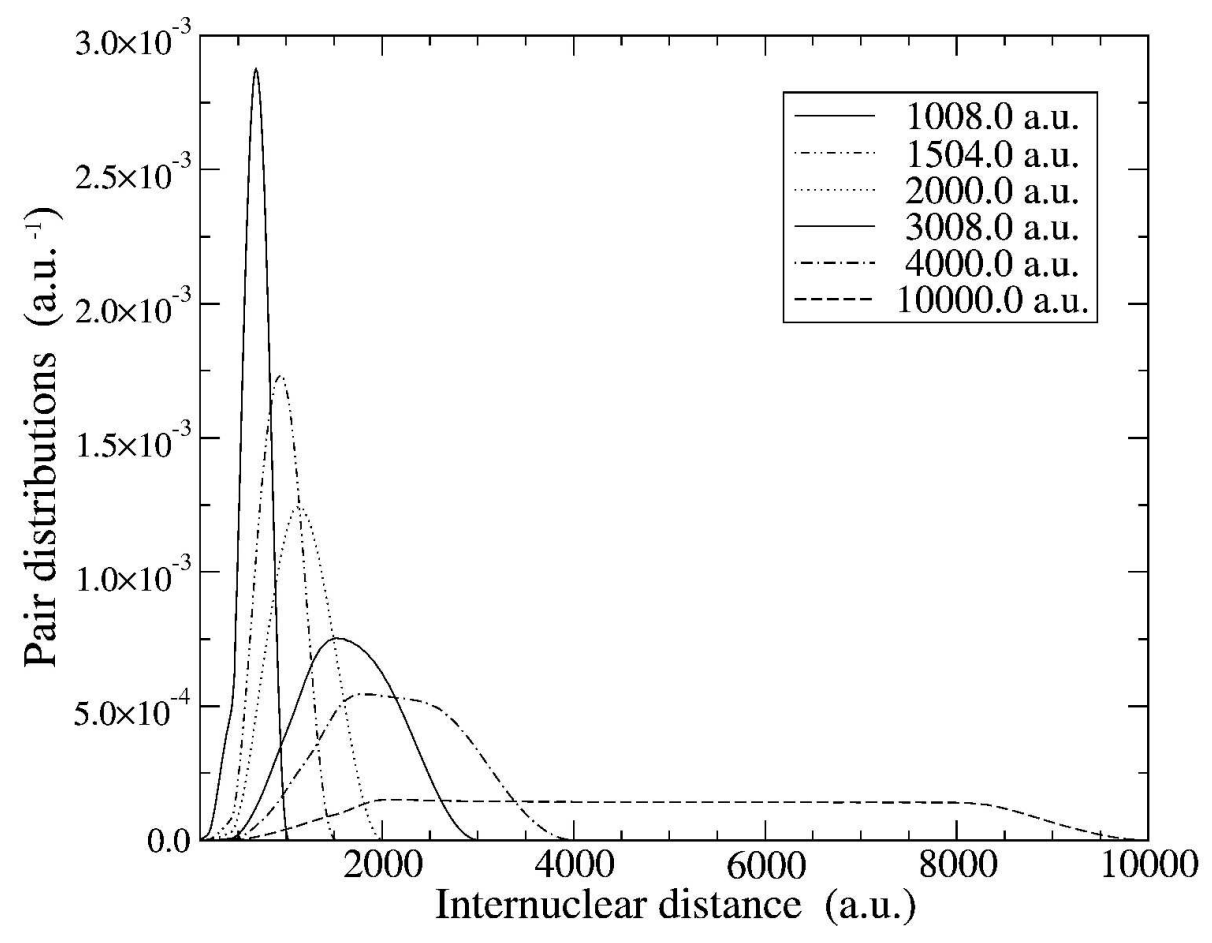

$279 \times 215 \mathrm{~mm}(600 \times 600 \mathrm{DPI})$ 

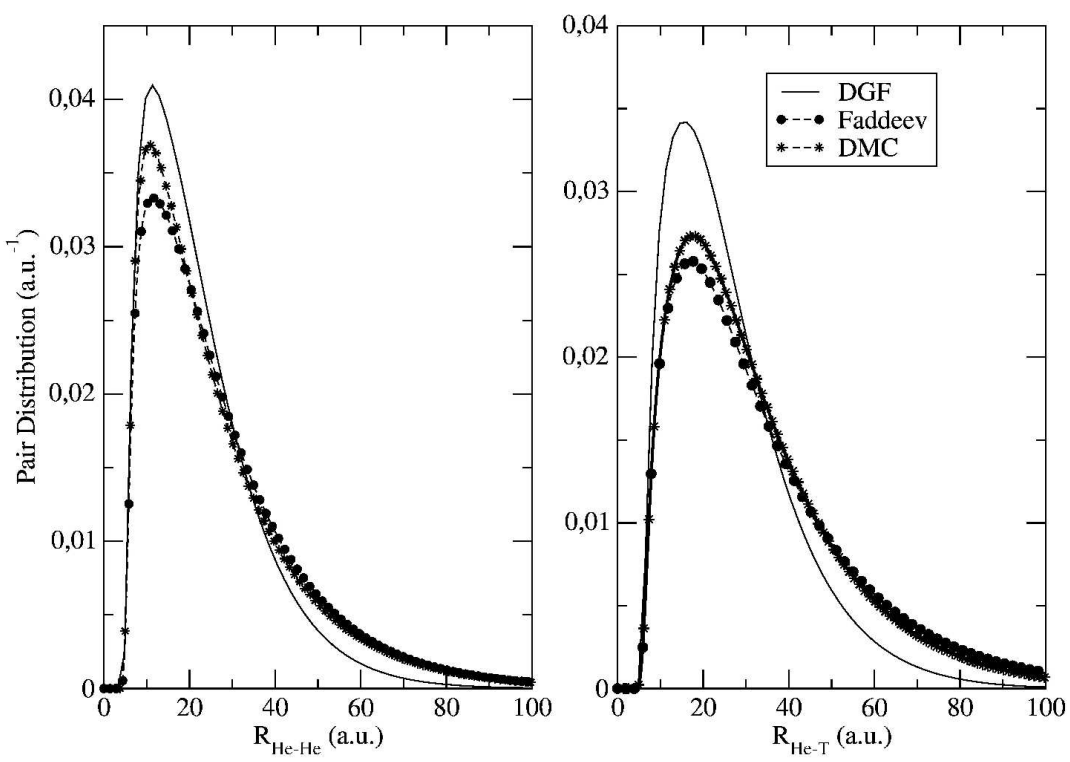

$297 \times 209 m m(600 \times 600$ DPI $)$ 


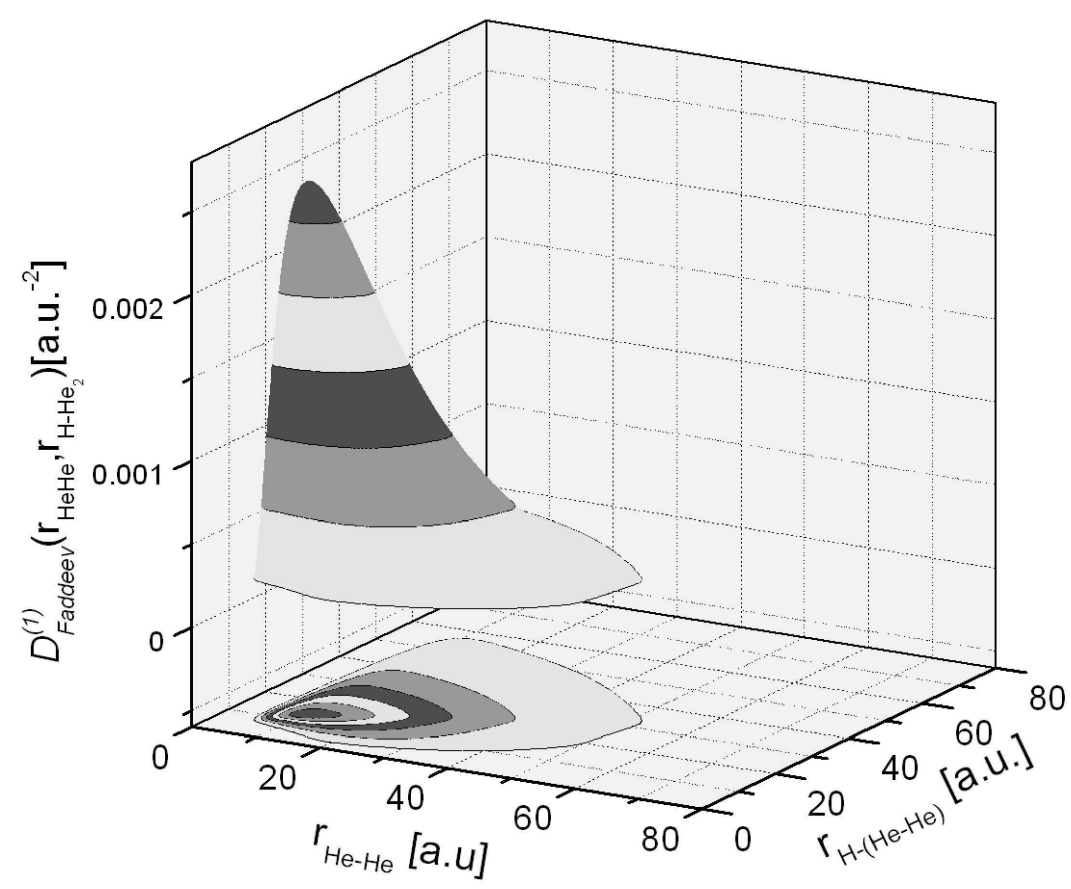

$114 \times 94 \mathrm{~mm}(600 \times 600 \mathrm{DPI})$ 


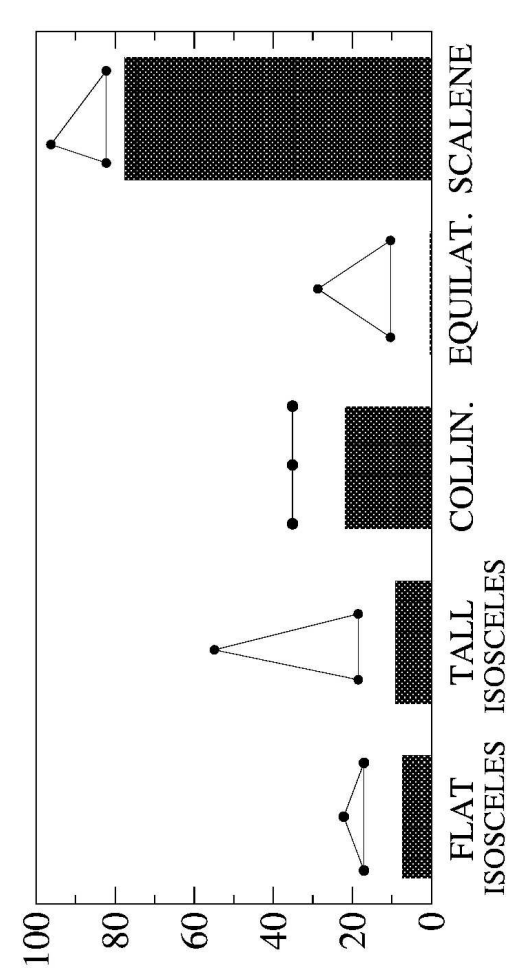

(\%) sไપชิเอM-opnəs $\mathbf{d}$

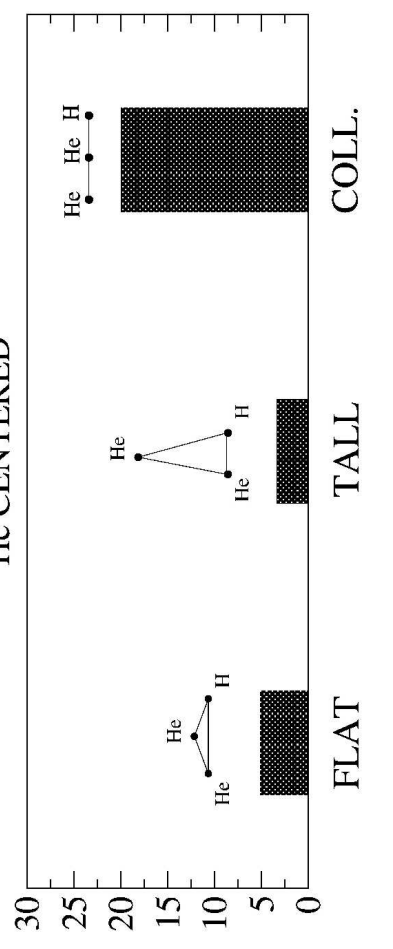

(\%) งłบฉ̊เฺM-opnəs

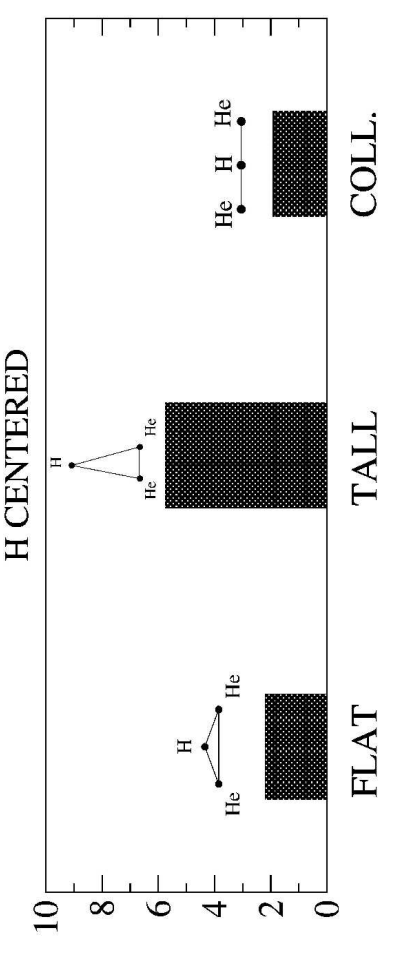

(\%) งฺบชิเฺม-opnəs

$320 \times 244 \mathrm{~mm}(600 \times 600$ DPI $)$ 


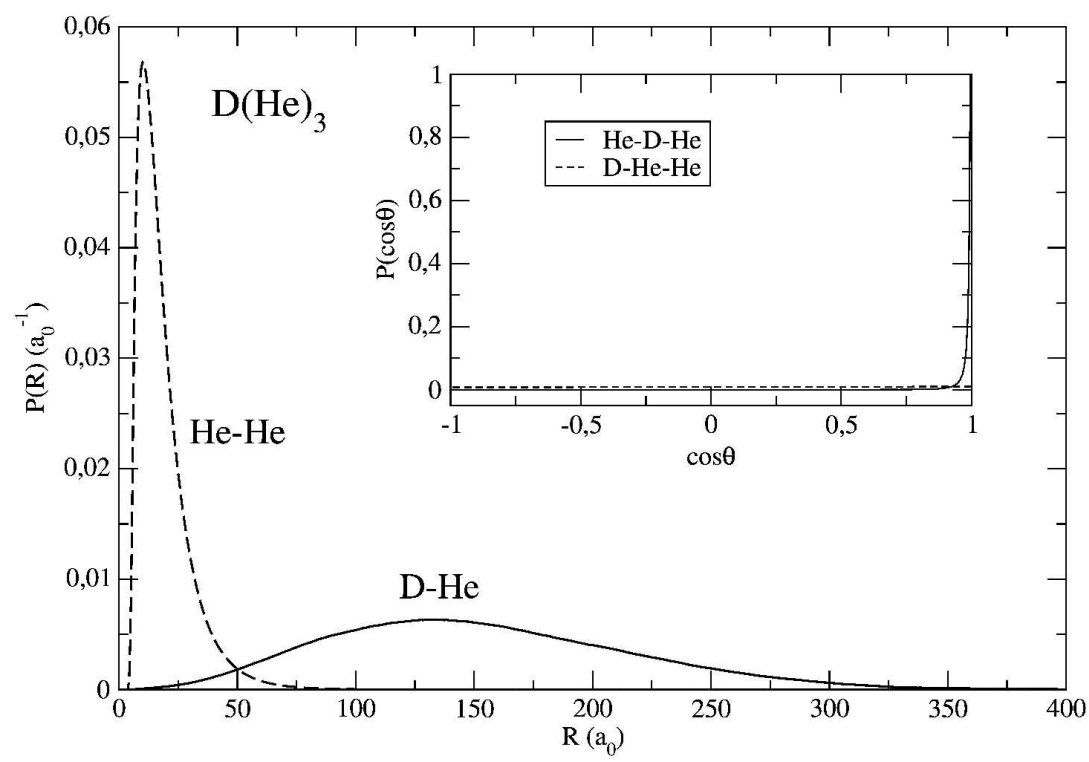

$297 \times 209 \mathrm{~mm}(600 \times 600 \mathrm{DPI})$ 


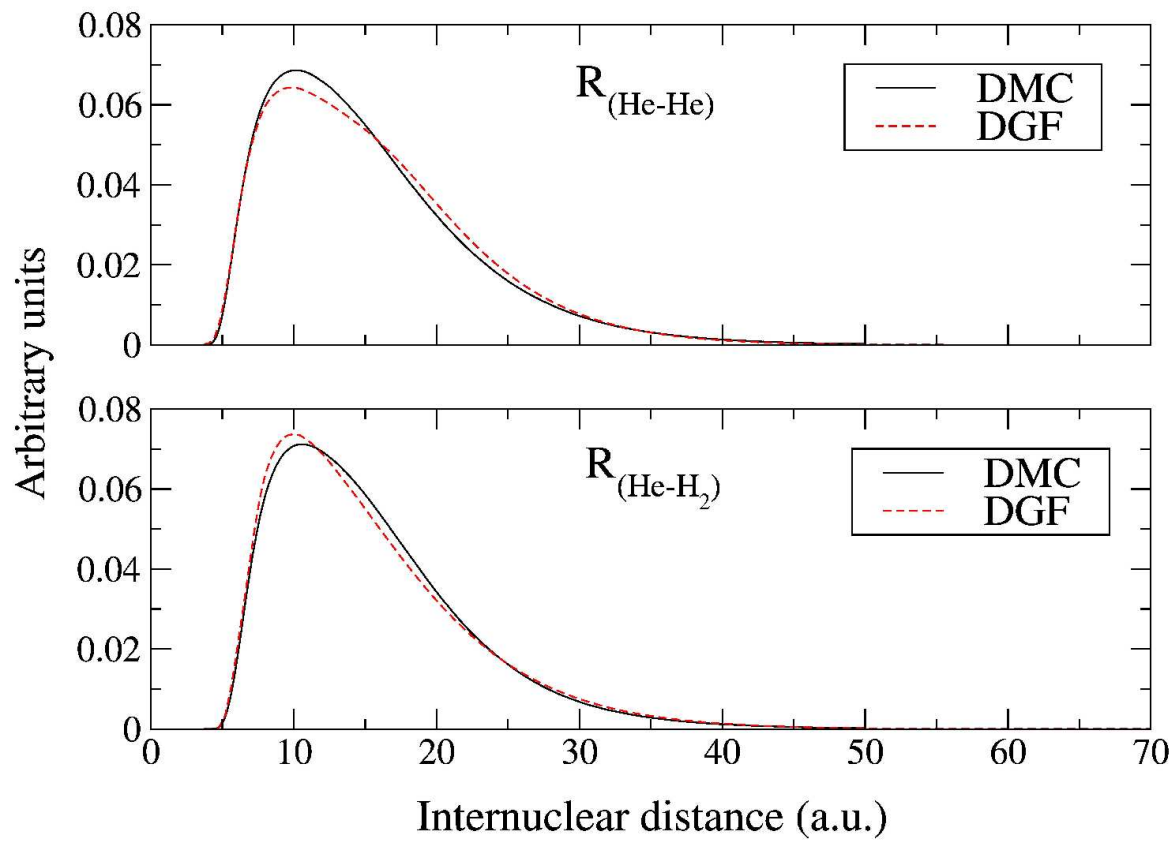

$279 \times 215 \mathrm{~mm}(600 \times 600 \mathrm{DPI})$ 